Pure and Applied Mathematics Quarterly

Volume 6, Number 2

(Special Issue: In honor of

Michael Atiyah and Isadore Singer)

$331-359,2010$

\title{
Hyperbolic Geometric Flow (I): Short-time Existence and Nonlinear Stability
}

\author{
Wen-Rong Dai, De-Xing Kong, Kefeng Liu
}

\begin{abstract}
In this paper we establish the short-time existence and uniqueness theorem for hyperbolic geometric flow, and prove the nonlinear stability of hyperbolic geometric flow defined on the Euclidean space with dimension larger than 4 . Wave equations satisfied by the curvatures are derived. The relations of the hyperbolic geometric flow with the Einstein equations and the Ricci flow are discussed.
\end{abstract}

Key words and phrases: hyperbolic geometric flow, quasilinear hyperbolic equation, strict hyperbolicity, symmetric hyperbolic system, nonlinear stability.

2000 Mathematics Subject Classification: 58J45, 58J47.

\section{INTRODUCTION}

This is the first of a series of papers devoted to the study of hyperbolic geometric flow and its applications to geometry and physics. Hyperbolic geometric flow was first studied by Kong and Liu in [11]. To introduce such flow we were partially motivated by the Einstein equations in general relativity and the recent progress in the Hamilton's Ricci flow, and by the possibility of applying the powerful theory of hyperbolic partial differential equations to geometry. Hyperbolic geometric flow is a system of nonlinear evolution partial differential equations of second order, it is very natural to understand certain wave phenomena in nature

Received June 12, 2007. 
as well as the geometry of manifolds, in particular, it describes the wave character of the metrics and curvatures of manifolds. We will see that the hyperbolic geometric flow carries many interesting features of both the Ricci flow as well as the Einstein equations. It has many promising applications to both subjects.

The elliptic and parabolic partial differential equations have been successfully applied to differential geometry and physics. Typical examples are the Hamilton's Ricci flow and Schoen-Yau's solution of the positive mass conjecture. A natural and important question is if we can apply the well-developed theory of hyperbolic differential equations to solve problems in differential geometry and theoretical physics. This series of papers is an attempt to apply the hyperbolic equation techniques to study some geometrical problems and physical problems. One has found interesting results in these directions, see for example [16] for the applications of the hyperbolic geometric flow equations to physics. Our results already show that the hyperbolic geometric flow is a natural and powerful tool to study some important problems arising from differential geometry and general relativity such as singularities, existence and regularity. In this paper we study the basic properties of the hyperbolic geometric flow such as the short-time existence, nonlinear stability and the wave feature of the curvatures. In the sequel we will study several fundamental problems, for example, long-time existence, formation of singularities, as well as the physical and geometrical applications.

Let $\mathscr{M}$ be an $n$-dimensional complete Riemannian manifold with Riemannian metric $g_{i j}$, the Levi-Civita connection is given by the Christoffel symbols

$$
\Gamma_{i j}^{k}=\frac{1}{2} g^{k l}\left\{\frac{\partial g_{j l}}{\partial x^{i}}+\frac{\partial g_{i l}}{\partial x^{j}}-\frac{\partial g_{i j}}{\partial x^{l}}\right\}
$$

where $g^{i j}$ is the inverse of $g_{i j}$. The Riemannian curvature tensors read

$$
R_{i j l}^{k}=\frac{\partial \Gamma_{j l}^{k}}{\partial x^{i}}-\frac{\partial \Gamma_{i l}^{k}}{\partial x^{j}}+\Gamma_{i p}^{k} \Gamma_{j l}^{p}-\Gamma_{j p}^{k} \Gamma_{i l}^{p}, \quad R_{i j k l}=g_{k p} R_{i j l}^{p} .
$$

The Ricci tensor is the contraction

$$
R_{i k}=g^{j l} R_{i j k l}
$$

and the scalar curvature is

$$
R=g^{i j} R_{i j}
$$


The hyperbolic geometric flow under the consideration is the following evolution equation

$$
\frac{\partial^{2} g_{i j}}{\partial t^{2}}=-2 R_{i j}
$$

for a family of Riemannian metrics $g_{i j}(t)$ on $\mathscr{M}$. More general hyperbolic geometric flows were also introduced in [11]. A natural and fundamental problem is the short-time existence and uniqueness theorem of hyperbolic geometric flow (1.1). In the present paper, we prove the following short-time existence and uniqueness theorem, the nonlinear stability theorem for Euclidean space, and derive the corresponding wave equations for the curvatures. These results were announced in Kong and Liu [11].

Theorem 1.1. Let $\left(\mathscr{M}, g_{i j}^{0}(x)\right)$ be a compact Riemannian manifold. Then there exists a constant $h>0$ such that the initial value problem

$$
\left\{\begin{array}{l}
\frac{\partial^{2}}{\partial t^{2}} g_{i j}(x, t)=-2 R_{i j}(x, t), \\
g_{i j}(x, 0)=g_{i j}^{0}(x), \frac{\partial g_{i j}}{\partial t}(x, 0)=k_{i j}^{0}(x),
\end{array}\right.
$$

has a unique smooth solution $g_{i j}(x, t)$ on $\mathscr{M} \times[0, h]$, where $k_{i j}^{0}(x)$ is a symmetric tensor on $\mathscr{M}$.

The main difficulty to prove this theorem is that, the hyperbolic geometric flow (1.1) is a system of nonlinear weakly-hyperbolic partial differential equations of second order. The degeneracy of the system is caused by the diffeomorphism group of $\mathscr{M}$ which acts as the gauge group of the hyperbolic geometric flow. Because the hyperbolic geometric flow (1.1) is only weakly hyperbolic, the shorttime existence and uniqueness result on a compact manifold does not come from the standard PDEs theory directly. In order to prove the above short-time existence and uniqueness theorem, using the gauge fixing idea as in the Ricci flow, we can derive a system of nonlinear strictly-hyperbolic partial differential equations of second order, thus Theorem 1.1 comes from the standard PDEs theory. On the other hand, we can reduce the hyperbolic geometric flow (1.1) to a quasilinear symmetric hyperbolic system of first order, then using the Friedrich's theory [5] of symmetric hyperbolic system (more exactly, the quasilinear version [3]) we can also prove Theorem 1.1.

Noting an important result on nonlinear wave equations (see [10]), we will find its other interesting application to geometry, by applying this result to the 
wave equations of curvatures. In the present paper we first use it to prove the nonlinear stability of the flat solution of the hyperbolic geometric flow defined on the Euclidean space with dimension larger than 4. More precisely, we have

Theorem 1.2. The flat metric $g_{i j}=\delta_{i j}$ of the Euclidean space $\mathbb{R}^{n}$ with $n \geq 5$ is nonlinearly stable.

See Section 4 for the precise definition of nonlinear stability which is very important in general relativity. The key point of the proof of this theorem is the global existence of classical solutions of the Cauchy problem for the nonlinear wave equations.

Similar to Hamilton [6], we derive the corresponding wave equations for the curvatures, for example, we have

Theorem 1.3. Under the hyperbolic geometric flow (1.1), the curvature tensor satisfies the evolution equation

$$
\begin{aligned}
\frac{\partial^{2}}{\partial t^{2}} R_{i j k l}= & \triangle R_{i j k l}+2\left(B_{i j k l}-B_{i j l k}-B_{i l j k}+B_{i k j l}\right) \\
& -g^{p q}\left(R_{p j k l} R_{q i}+R_{i p k l} R_{q j}+R_{i j p l} R_{q k}+R_{i j k p} R_{q l}\right) \\
& +2 g_{p q}\left(\frac{\partial \Gamma_{i l}^{p}}{\partial t} \frac{\partial \Gamma_{j k}^{q}}{\partial t}-\frac{\partial \Gamma_{j l}^{p}}{\partial t} \frac{\partial \Gamma_{i k}^{q}}{\partial t}\right),
\end{aligned}
$$

where $B_{i j k l}=g^{p r} g^{q s} R_{p i q j} R_{r k s l}$ and $\triangle$ is the Laplacian with respect to the evolving metric.

The wave equations for the Ricci and scalar curvatures are stated and proved in Section 5. This is similar to the Ricci flow equation, except that there are quadratic lower order terms involving the connection coefficients. It turns out that there is a very rich theory in nonlinear wave equations to deal with such terms, see [10].

From the above results we can already see that the hyperbolic geometric flow has many features of the Ricci flow, therefore many well-developed techniques in the Ricci flow may be applied to the study of this new kind of flow equations. On the other hand, the hyperbolic geometric flow can also be viewed as the leading terms of the vacuum Einstein equations. Since the hyperbolic geometric flow contains the major terms in the Einstein equations, it not only becomes simpler 
and more symmetric, but also possesses rich and beautiful geometric properties. See Section 6 for more detailed discussions on the relations between the hyperbolic geometric flow and the Einstein equations, and more generally its relations with other important problems in general relativity.

The paper is organized as follows. In Section 2, using the gauge fixing idea as in the Ricci flow, we derive a system of nonlinear strictly-hyperbolic partial differential equations of second order. In Section 3 we reduce the hyperbolic geometric flow (1.1) to a quasilinear symmetric hyperbolic system of first order, and give the proof of Theorem 1.1. Section 4 is devoted to the nonlinear stability of the hyperbolic geometric flow defined on the Euclidean space with dimension larger than 4. In Section 5, we derive the wave equations satisfied by the curvatures, and illustrate the wave character of the curvatures. Some discussions are given in Section 6.

\section{Strict hyperbolicity of hyperbolic GeOMEtric Flow}

In this section we consider a modified system of evolution equations of the hyperbolic geometric flow, which is strictly hyperbolic so that we can get a solution for a short time by solving the corresponding Cauchy problem. The solution of the system (1.1) then comes from the solution of the modified equations.

Let $\mathscr{M}$ be a compact $n$-dimensional manifold. We consider the hyperbolic geometric flow (1.1) on $\mathscr{M}$, that is,

$$
\frac{\partial^{2}}{\partial t^{2}} g_{i j}(x, t)=-2 R_{i j}(x, t)
$$

Suppose $\hat{g}_{i j}(x, t)$ is a solution of the hyperbolic geometric flow (2.1), and $\psi_{t}$ : $\mathscr{M} \rightarrow \mathscr{M}$ is a family of diffeomorphisms of $\mathscr{M}$. Let

$$
g_{i j}(x, t)=\psi_{t}^{*} \hat{g}_{i j}(x, t)
$$

be the pull-back metrics. We now want to find the evolution equations for the metrics $g_{i j}(x, t)$. Denote by $y(x, t)=\varphi_{t}(x)=\left(y^{1}(x, t), y^{2}(x, t), \cdots, y^{n}(x, t)\right)$ in local coordinates. Then

$$
g_{i j}(x, t)=\frac{\partial y^{\alpha}}{\partial x^{i}} \frac{\partial y^{\beta}}{\partial x^{j}} \hat{g}_{\alpha \beta}(y, t)
$$


and

$$
\begin{aligned}
\frac{\partial}{\partial t} g_{i j}(x, t) & =\frac{\partial}{\partial t}\left[\hat{g}_{\alpha \beta}(y, t) \frac{\partial y^{\alpha}}{\partial x^{i}} \frac{\partial y^{\beta}}{\partial x^{j}}\right] \\
& =\frac{\partial y^{\alpha}}{\partial x^{i}} \frac{\partial y^{\beta}}{\partial x^{j}} \frac{d}{d t} \hat{g}_{\alpha \beta}(y(x, t), t)+\hat{g}_{\alpha \beta}(y, t) \frac{\partial}{\partial t}\left(\frac{\partial y^{\alpha}}{\partial x^{i}} \frac{\partial y^{\beta}}{\partial x^{j}}\right) .
\end{aligned}
$$

Furthermore, we have

$$
\begin{aligned}
\frac{\partial^{2}}{\partial t^{2}} g_{i j}(x, t)= & \frac{\partial y^{\alpha}}{\partial x^{i}} \frac{\partial y^{\beta}}{\partial x^{j}} \frac{d^{2} \hat{g}_{\alpha \beta}}{d t^{2}}(y(x, t), t)+\frac{\partial}{\partial x^{i}}\left(\frac{\partial^{2} y^{\alpha}}{\partial t^{2}}\right) \frac{\partial y^{\beta}}{\partial x^{j}} \hat{g}_{\alpha \beta} \\
& +\frac{\partial y^{\alpha}}{\partial x^{i}} \frac{\partial}{\partial x^{j}}\left(\frac{\partial^{2} y^{\beta}}{\partial t^{2}}\right) \hat{g}_{\alpha \beta}+2 \frac{\partial}{\partial x^{i}}\left(\frac{\partial y^{\alpha}}{\partial t}\right) \frac{\partial y^{\beta}}{\partial x^{j}} \frac{d \hat{g}_{\alpha \beta}}{d t} \\
& +2 \frac{\partial y^{\alpha}}{\partial x^{i}} \frac{\partial}{\partial x^{j}}\left(\frac{\partial y^{\beta}}{\partial t}\right) \frac{d \hat{g}_{\alpha \beta}}{d t}+2 \frac{\partial}{\partial x^{i}}\left(\frac{\partial y^{\alpha}}{\partial t}\right) \frac{\partial}{\partial x^{j}}\left(\frac{\partial y^{\beta}}{\partial t}\right) \hat{g}_{\alpha \beta} .
\end{aligned}
$$

On the other hand,

$$
\begin{gathered}
\frac{d \hat{g}_{\alpha \beta}}{d t}(y(x, t), t)=\frac{\partial \hat{g}_{\alpha \beta}}{\partial y^{\gamma}} \frac{\partial y^{\gamma}}{\partial t}+\frac{\partial \hat{g}_{\alpha \beta}}{\partial t} \\
\frac{d^{2} \hat{g}_{\alpha \beta}}{d t^{2}}(y(x, t), t)=\frac{\partial^{2} \hat{g}_{\alpha \beta}}{\partial y^{\gamma} \partial y^{\lambda}} \frac{\partial y^{\gamma}}{\partial t} \frac{\partial y^{\lambda}}{\partial t}+2 \frac{\partial^{2} \hat{g}_{\alpha \beta}}{\partial y^{\gamma} \partial t} \frac{\partial y^{\gamma}}{\partial t}+\frac{\partial^{2} \hat{g}_{\alpha \beta}}{\partial t^{2}}+\frac{\partial \hat{g}_{\alpha \beta}}{\partial y^{\gamma}} \frac{\partial^{2} y^{\gamma}}{\partial t^{2}}
\end{gathered}
$$

and

$$
\frac{\partial^{2} \hat{g}_{\alpha \beta}}{\partial t^{2}}(y, t)=-2 \hat{R}_{\alpha \beta}(y, t) .
$$

It follows from (2.3) that

$$
\begin{aligned}
\frac{\partial^{2} g_{i j}}{\partial t^{2}}(x, t)= & -2 \hat{R}_{\alpha \beta}(y, t) \frac{\partial y^{\alpha}}{\partial x^{i}} \frac{\partial y^{\beta}}{\partial x^{j}}+\frac{\partial^{2} \hat{g}_{\alpha \beta}}{\partial y^{\gamma} \partial y^{\lambda}} \frac{\partial y^{\alpha}}{\partial x^{i}} \frac{\partial y^{\beta}}{\partial x^{j}} \frac{\partial y^{\gamma}}{\partial t} \frac{\partial y^{\lambda}}{\partial t} \\
& +2 \frac{\partial^{2} \hat{g}_{\alpha \beta}}{\partial y^{\gamma} \partial t} \frac{\partial y^{\alpha}}{\partial x^{i}} \frac{\partial y^{\beta}}{\partial x^{j}} \frac{\partial y^{\gamma}}{\partial t}+\frac{\partial}{\partial x^{i}}\left(\hat{g}_{\alpha \beta} \frac{\partial y^{\beta}}{\partial x^{j}} \frac{\partial^{2} y^{\alpha}}{\partial t^{2}}\right)+\frac{\partial}{\partial x^{j}}\left(\hat{g}_{\alpha \beta} \frac{\partial y^{\beta}}{\partial x^{i}} \frac{\partial^{2} y^{\alpha}}{\partial t^{2}}\right) \\
& +\left[\frac{\partial \hat{g}_{\alpha \beta}}{\partial y^{\gamma}} \frac{\partial y^{\alpha}}{\partial x^{i}} \frac{\partial y^{\beta}}{\partial x^{j}}-\frac{\partial}{\partial x^{i}}\left(\frac{\partial y^{\beta}}{\partial x^{j}} \hat{g}_{\beta \gamma}\right)-\frac{\partial}{\partial x^{j}}\left(\frac{\partial y^{\beta}}{\partial x^{i}} \hat{g}_{\beta \gamma}\right)\right] \frac{\partial^{2} y^{\gamma}}{\partial t^{2}} \\
& +2 \frac{\partial}{\partial x^{i}}\left(\frac{\partial y^{\alpha}}{\partial t}\right) \frac{\partial y^{\beta}}{\partial x^{j}}\left(\frac{\partial \hat{g}_{\alpha \beta}}{\partial t}+\frac{\partial \hat{g}_{\alpha \beta}}{\partial y^{\gamma}} \frac{\partial y^{\gamma}}{\partial t}\right) \\
& +2 \frac{\partial y^{2}}{\partial x^{i}} \frac{\partial}{\partial x^{j}}\left(\frac{\partial y^{\beta}}{\partial t}\right)\left(\frac{\partial \hat{g}_{\alpha \beta}}{\partial y^{\gamma}} \frac{\partial y^{\gamma}}{\partial t}+\frac{\partial \hat{g}_{\alpha \beta}}{\partial t}\right) \\
& +2 \hat{g}_{\alpha \beta} \frac{\partial}{\partial x^{i}}\left(\frac{\partial y^{\alpha}}{\partial t}\right) \frac{\partial}{\partial x^{j}}\left(\frac{\partial y^{\beta}}{\partial t}\right) .
\end{aligned}
$$


Let us choose the normal coordinates $\left\{x^{i}\right\}$ around a fixed point $p \in \mathscr{M}$ such that $\frac{\partial g_{i j}}{\partial x^{k}}=0$ at $p$. We next prove that, at $p \in \mathscr{M}$,

$$
\frac{\partial \hat{g}_{\alpha \beta}}{\partial y^{\gamma}} \frac{\partial y^{\alpha}}{\partial x^{i}} \frac{\partial y^{\beta}}{\partial x^{j}}-\frac{\partial}{\partial x^{i}}\left(\frac{\partial y^{\beta}}{\partial x^{j}} \hat{g}_{\beta \gamma}\right)-\frac{\partial}{\partial x^{j}}\left(\frac{\partial y^{\beta}}{\partial x^{i}} \hat{g}_{\beta \gamma}\right)=0, \quad \forall i, j, \gamma=1, \cdots, n \text {. }
$$

The left hand side of (2.5) is

$$
\begin{aligned}
& \frac{\partial \hat{g}_{\alpha \beta}}{\partial y^{\gamma}} \frac{\partial y^{\alpha}}{\partial x^{i}} \frac{\partial y^{\beta}}{\partial x^{j}}-\frac{\partial}{\partial x^{i}}\left(\frac{\partial y^{\beta}}{\partial x^{j}} \hat{g}_{\beta \gamma}\right)-\frac{\partial}{\partial x^{j}}\left(\frac{\partial y^{\beta}}{\partial x^{i}} \hat{g}_{\alpha \beta}\right) \\
= & \frac{\partial}{\partial y^{\gamma}}\left(g_{m n} \frac{\partial x^{m}}{\partial y^{\alpha}} \frac{\partial x^{n}}{\partial y^{\beta}}\right) \frac{\partial y^{\alpha}}{\partial x^{i}} \frac{\partial y^{\beta}}{\partial x^{j}}-\frac{\partial}{\partial x^{i}}\left(g_{m j} \frac{\partial x^{m}}{\partial y^{\gamma}}\right)-\frac{\partial}{\partial x^{j}}\left(g_{m i} \frac{\partial x^{m}}{\partial y^{\gamma}}\right) \\
= & g_{m n}\left[\frac{\partial}{\partial y^{\gamma}}\left(\frac{\partial x^{m}}{\partial y^{\alpha}}\right) \frac{\partial x^{n}}{\partial y^{\beta}} \frac{\partial y^{\alpha}}{\partial x^{i}} \frac{\partial y^{\beta}}{\partial x^{j}}+\frac{\partial x^{m}}{\partial y^{\alpha}} \frac{\partial}{\partial y^{\gamma}}\left(\frac{\partial x^{n}}{\partial y^{\beta}}\right) \frac{\partial y^{\alpha}}{\partial x^{i}} \frac{\partial y^{\beta}}{\partial x^{j}}\right] \\
& -g_{m j} \frac{\partial}{\partial x^{i}}\left(\frac{\partial x^{m}}{\partial y^{\gamma}}\right)-g_{m i} \frac{\partial}{\partial x^{j}}\left(\frac{\partial x^{m}}{\partial y^{\gamma}}\right) \\
= & g_{m j} \frac{\partial}{\partial y^{\gamma}}\left(\frac{\partial x^{m}}{\partial y^{\alpha}}\right) \frac{\partial y^{\alpha}}{\partial x^{i}}+g_{m i} \frac{\partial}{\partial y^{\gamma}}\left(\frac{\partial x^{m}}{\partial y^{\beta}}\right) \frac{\partial y^{\beta}}{\partial x^{j}} \\
& -g_{m j} \frac{\partial}{\partial x^{i}}\left(\frac{\partial x^{m}}{\partial y^{\gamma}}\right)-g_{m i} \frac{\partial}{\partial x^{j}}\left(\frac{\partial x^{m}}{\partial y^{\gamma}}\right) \\
= & g_{m j} \frac{\partial}{\partial y^{\alpha}}\left(\frac{\partial x^{m}}{\partial y^{\gamma}}\right) \frac{\partial y^{\alpha}}{\partial x^{i}}+g_{m i} \frac{\partial}{\partial y^{\beta}}\left(\frac{\partial x^{m}}{\partial y^{\gamma}}\right) \frac{\partial y^{\beta}}{\partial x^{j}} \\
& -g_{m j} \frac{\partial}{\partial x^{i}}\left(\frac{\partial x^{m}}{\partial y^{\gamma}}\right)-g_{m i} \frac{\partial}{\partial x^{j}}\left(\frac{\partial x^{m}}{\partial y^{\gamma}}\right) \\
= & 0 .
\end{aligned}
$$

So (2.5) holds.

By (2.4) and (2.5), we have

$$
\begin{aligned}
\frac{\partial^{2} g_{i j}}{\partial t^{2}}(x, t)= & -2 R_{i j}(x, t)+\frac{\partial}{\partial x^{i}}\left(g_{m j} \frac{\partial x^{m}}{\partial y^{\alpha}} \frac{\partial^{2} y^{\alpha}}{\partial t^{2}}\right)+\frac{\partial}{\partial x^{j}}\left(g_{m i} \frac{\partial y^{\alpha}}{\partial x^{m}} \frac{\partial^{2} y^{\alpha}}{\partial t^{2}}\right) \\
& +\frac{\partial^{2} \hat{g}_{\alpha \beta}}{\partial y^{\gamma} \partial y^{\lambda}} \frac{\partial y^{\alpha}}{\partial x^{i}} \frac{\partial y^{\beta}}{\partial x^{j}} \frac{\partial y^{\gamma}}{\partial t} \frac{\partial y^{\lambda}}{\partial t}+2 \frac{\partial \hat{g}_{\alpha \beta}}{\partial y^{\gamma} \partial t} \frac{\partial y^{\gamma}}{\partial t} \frac{\partial y^{\alpha}}{\partial x^{i}} \frac{\partial y^{\beta}}{\partial x^{j}} \\
& +2 \frac{\partial}{\partial x^{i}}\left(\frac{\partial y^{\alpha}}{\partial t}\right) \frac{\partial y^{\beta}}{\partial x^{j}}\left(\frac{\partial \widehat{g}_{\alpha \beta}}{\partial t}+\frac{\partial \widehat{g}_{\alpha \beta}}{\partial y^{\gamma}} \frac{\partial y^{\gamma}}{\partial t}\right) \\
& +2 \frac{\partial y^{\alpha}}{\partial x^{i}} \frac{\partial}{\partial x^{j}}\left(\frac{\partial y^{\beta}}{\partial t}\right)\left(\frac{\partial \widehat{g}_{\alpha \beta}}{\partial t}+\frac{\partial \widehat{g}_{\alpha \beta}}{\partial y^{\gamma}} \frac{\partial y^{\gamma}}{\partial t}\right) \\
& +2 \widehat{g}_{\alpha \beta} \frac{\partial}{\partial x^{i}}\left(\frac{\partial y^{\alpha}}{\partial t}\right) \frac{\partial}{\partial x^{j}}\left(\frac{\partial y^{\beta}}{\partial t}\right) .
\end{aligned}
$$


We define $y(x, t)=\varphi_{t}(x)$ by the following initial value problem

$$
\left\{\begin{array}{l}
\frac{\partial^{2} y^{\alpha}}{\partial t^{2}}=\frac{\partial y^{\alpha}}{\partial x^{k}} g^{j l}\left(\Gamma_{j l}^{k}-\stackrel{\circ}{\Gamma_{j l}^{k}}\right), \\
y^{\alpha}(x, 0)=x^{\alpha}, \quad \frac{\partial}{\partial t} y^{\alpha}(x, 0)=y_{1}^{\alpha}(x)
\end{array}\right.
$$

and define the vector filed

$$
V_{i}=g_{i k} g^{j l}\left(\Gamma_{j l}^{k}-\stackrel{\circ}{\Gamma^{k}}\right)
$$

where $\Gamma_{j l}^{k}$ and $\stackrel{\circ}{\Gamma}_{j l}^{k}$ are the connection coefficients corresponding to the metrics $g_{i j}(x, t)$ and $g_{i j}(x, 0)$, respectively, $y_{1}^{\alpha}(x) \quad(\alpha=1,2, \cdots, n)$ are arbitrary $C^{\infty}$ smooth functions on the manifold $\mathscr{M}$. We get the following evolution equation for the pull-back metric

$$
\left\{\begin{aligned}
\frac{\partial^{2} g_{i j}}{\partial t^{2}}(x, t)= & -2 R_{i j}(x, t)+\nabla_{i} V_{j}+\nabla_{j} V_{i}+\frac{\partial^{2} \widehat{g}_{\alpha \beta}}{\partial y^{\gamma} \partial y^{\lambda}} \frac{\partial y^{\alpha}}{\partial x^{i}} \frac{\partial y^{\beta}}{\partial x^{j}} \frac{\partial y^{\gamma}}{\partial t} \frac{\partial y^{\lambda}}{\partial t} \\
& +2 \frac{\partial^{2} \widehat{g}_{\alpha \beta}}{\partial y^{\gamma} \partial t} \frac{\partial y^{\alpha}}{\partial x^{i}} \frac{\partial y^{\beta}}{\partial x^{j}} \frac{\partial y^{\gamma}}{\partial t}+2 \frac{\partial}{\partial x^{i}}\left(\frac{\partial y^{\alpha}}{\partial t}\right) \frac{\partial y^{\beta}}{\partial x^{j}}\left(\frac{\partial \widehat{g}_{\alpha \beta}}{\partial t}+\frac{\partial \widehat{g}_{\alpha \beta}}{\partial y^{\gamma}} \frac{\partial y^{\gamma}}{\partial t}\right) \\
& +2 \frac{\partial}{\partial x^{j}}\left(\frac{\partial y^{\beta}}{\partial t}\right) \frac{\partial y^{\alpha}}{\partial x^{i}}\left(\frac{\partial \widehat{g}_{\alpha \beta}}{\partial t}+\frac{\partial \widehat{g}_{\alpha \beta}}{\partial y^{\gamma}} \frac{\partial y^{\gamma}}{\partial t}\right) \\
& +2 \widehat{g}_{\alpha \beta} \frac{\partial}{\partial x^{i}}\left(\frac{\partial y^{\alpha}}{\partial t}\right) \frac{\partial}{\partial x^{j}}\left(\frac{\partial y^{\beta}}{\partial t}\right) \\
\triangleq & -2 R_{i j}(x, t)+\nabla_{i} V_{j}+\nabla_{j} V_{i}+F\left(D y, D_{t} D_{x} y\right), \\
= & g_{i j}^{0}(x), \quad \frac{\partial}{\partial t} g_{i j}(x, 0)=k_{i j}^{0}(x),
\end{aligned}\right.
$$

where

$$
D y=\left(\frac{\partial y^{\alpha}}{\partial t}, \frac{\partial y^{\alpha}}{\partial x^{i}}\right), \quad D_{t} D_{x} y=\left(\frac{\partial^{2} y^{\alpha}}{\partial x^{i} \partial t}\right) \quad(\alpha, i=1,2, \cdots, n) .
$$

Let

$$
\widehat{\lambda}=\left(\frac{\partial y^{\alpha}}{\partial t}, \frac{\partial y^{\alpha}}{\partial x^{i}}, \frac{\partial^{2} y^{\alpha}}{\partial x^{i} \partial t}\right) \quad(\alpha, i=1,2, \cdots, n) .
$$

The nonlinear term $F=F(\widehat{\lambda})=F\left(D y, D_{t} D_{x} y\right)$ in $(2.8)$ is smooth and $F(\widehat{\lambda})=$ $O\left(|\widehat{\lambda}|^{2}\right)$ holds.

Since

$$
\Gamma_{j i}^{k}=\frac{\partial y^{\alpha}}{\partial x^{j}} \frac{\partial y^{\beta}}{\partial x^{i}} \frac{\partial x^{k}}{\partial y^{\gamma}} \widehat{\Gamma}_{\alpha \beta}^{\gamma}+\frac{\partial x^{k}}{\partial y^{\alpha}} \frac{\partial^{2} y^{\alpha}}{\partial x^{j} \partial x^{i}},
$$


the initial value problem (2.7) can be written as

$$
\left\{\begin{array}{l}
\frac{\partial^{2} y^{\alpha}}{\partial t^{2}}=g^{j l}\left(\frac{\partial^{2} y^{\alpha}}{\partial x^{j} \partial x^{l}}-\stackrel{\circ}{\Gamma_{j l}^{k}} \frac{\partial y^{\alpha}}{\partial x^{k}}+\widehat{\Gamma}_{\beta \gamma}^{\alpha} \frac{\partial y^{\beta}}{\partial x^{j}} \frac{\partial y^{\gamma}}{\partial x^{i}}\right) \\
y^{\alpha}(x, 0)=x^{\alpha}, \quad \frac{\partial}{\partial t} y^{\alpha}(x, 0)=y_{1}^{\alpha}(x) .
\end{array}\right.
$$

At the same time, in the normal coordinates $\left\{x^{i}\right\}$,

$$
\begin{aligned}
-2 R_{i j}(x, t)+ & \nabla_{i} V_{j}+\nabla_{j} V_{i}=\frac{\partial}{\partial x^{i}}\left\{g^{k l} \frac{\partial g_{k l}}{\partial x^{j}}\right\}-\frac{\partial}{\partial x^{k}}\left\{g^{k l}\left(\frac{\partial g_{j l}}{\partial x^{i}}+\frac{\partial g_{i l}}{\partial x^{j}}-\frac{\partial g_{i j}}{\partial x^{l}}\right)\right\} \\
& +g_{j k} g^{p q} \frac{\partial}{\partial x^{i}}\left\{\frac{1}{2} g^{k l}\left(\frac{\partial g_{p l}}{\partial x^{q}}+\frac{\partial g_{q l}}{\partial x^{p}}-\frac{\partial g_{p q}}{\partial x^{l}}\right)\right\} \\
& +g_{i k} g^{p q} \frac{\partial}{\partial x^{j}}\left\{\frac{1}{2} g^{k l}\left(\frac{\partial g_{p l}}{\partial x^{q}} \frac{\partial g_{q l}}{\partial x^{p}}-\frac{\partial g_{p q}}{\partial x^{l}}\right)\right\}+(\text { lower order terms) } \\
= & g^{k l}\left\{\frac{\partial^{2} g_{k l}}{\partial x^{i} \partial x^{j}}-\frac{\partial^{2} g_{j l}}{\partial x^{i} \partial x^{k}}-\frac{\partial^{2} g_{i l}}{\partial x^{j} \partial x^{k}}+\frac{\partial^{2} g_{i j}}{\partial x^{k} \partial x^{l}}\right\} \\
& +\frac{1}{2} g^{p q}\left\{\frac{\partial^{2} g_{p j}}{\partial x^{i} \partial x^{q}}+\frac{\partial^{2} g_{q j}}{\partial x^{i} \partial x^{q}}-\frac{\partial^{2} g_{p q}}{\partial x^{i} \partial x^{j}}\right\} \\
& +\frac{1}{2} g^{p q}\left\{\frac{\partial^{2} g_{p i}}{\partial x^{i} \partial x^{q}}+\frac{\partial^{2} g_{q i}}{\partial x^{j} \partial x^{p}}-\frac{\partial^{2} g_{p q}}{\partial x^{i} \partial x^{j}}\right\}+\text { (lower order terms) } \\
= & g^{k l} \frac{\partial^{2} g_{i j}}{\partial x^{k} \partial x^{l}}+(\text { lower order terms). }
\end{aligned}
$$

Thereby, the initial value problem (2.8) can be written as

$$
\left\{\begin{array}{l}
\frac{\partial^{2} g_{i j}}{\partial t^{2}}(x, t)=g^{k l} \frac{\partial^{2} g_{i j}}{\partial x^{k} \partial x^{l}}+F\left(D y, D_{t} D_{x} y\right)+G\left(g, D_{x} g\right), \\
g_{i j}(x, 0)=g_{i j}^{0}(x), \quad \frac{\partial}{\partial t} g_{i j}(x, 0)=k_{i j}^{0}(x)
\end{array}\right.
$$

where $g=\left(g_{i j}\right), D_{x} g=\left(\frac{\partial g_{i j}}{\partial x^{k}}\right)(i, j, k=1,2, \cdots, n)$. Let

$$
\widehat{\mu}=\left(g_{i j}, \frac{\partial g_{i j}}{\partial x^{k}}\right) \quad(i, j, k=1,2, \cdots, n) .
$$

The nonlinear term $G=G(\widehat{\mu})=G\left(g, D_{x} g\right)$ in (2.10) is smooth and quadratic with respect to $D_{x} g$.

We observe that both (2.9) and (2.10) are clearly strictly hyperbolic systems. Since the equations (2.9) and (2.10) are strictly hyperbolic and the manifold $\mathscr{M}$ is compact, it follows from the standard theory of hyperbolic equations (see [8], 
[9], [10]) that the system united by (2.9) and (2.10) has a unique smooth solution for a short time. Thus, we have proved Theorem 1.1.

\section{Symmetrization of hyperbolic Geometric Flow - SECOND Proof OF THEOREM 1.1}

In this section we reduce the hyperbolic geometric flow (2.1) to a symmetric hyperbolic system. Then we use the theory of symmetric hyperbolic system to give another proof of Theorem 1.1.

Let $\mathscr{M}$ be a compact $n$-dimensional manifold and $g_{i j}(x, t)$ is a hyperbolic geometric flow on $\mathscr{M}$. We denote the corresponding connection coefficients, the Riemannian curvature tensor and the Ricci curvature tensor by $\Gamma_{i j}^{k}, R_{i j k l}$ and $R_{i k}$, respectively.

We consider the space-time $\mathbb{R} \times \mathscr{M}$ with the Lorentzian metric

$$
d s^{2}=-d t^{2}+g_{i j}(x, t) d x^{i} d x^{j}
$$

and denote the corresponding connection coefficients, the Riemannian curvature tensor and the Ricci curvature tensor by $\widehat{\Gamma}_{\alpha \beta}^{\gamma}, \widehat{R}_{\alpha \beta \gamma \lambda}$ and $\widehat{R}_{\alpha \beta}$, respectively. Here and hereafter, the Greek indices run from 0 to $n$, Latin indices from 1 to $n$. The summation convention is employed. We also denote $x^{0}=t$.

By direct computations,

$$
\begin{aligned}
& \widehat{\Gamma}_{i j}^{k}=\Gamma_{i j}^{k}, \quad \widehat{\Gamma}_{i j}^{0}=\frac{1}{2} \frac{\partial g_{i j}}{\partial t}, \quad \widehat{\Gamma}_{0 l}^{k}=\widehat{\Gamma}_{l 0}^{k}=\frac{1}{2} g^{k i} \frac{\partial g_{i l}}{\partial t}, \quad \widehat{\Gamma}_{0 i}^{0}=0, \quad \widehat{\Gamma}_{00}^{i}=0, \quad \widehat{\Gamma}_{00}^{0}=0 \\
& \widehat{R}_{i j l}^{k}=\frac{\partial \Gamma_{j l}^{k}}{\partial x^{i}}-\frac{\partial \Gamma_{i l}^{k}}{\partial x^{j}}+\widehat{\Gamma}_{i \alpha}^{k} \widehat{\Gamma}_{j l}^{\alpha}-\widehat{\Gamma}_{j \alpha}^{k} \widehat{\Gamma}_{i l}^{\alpha}=R_{i j l}^{k}+\frac{1}{4} g^{k m} \frac{\partial g_{m i}}{\partial t} \frac{\partial g_{j l}}{\partial t}-\frac{1}{4} g^{k m} \frac{\partial g_{m j}}{\partial t} \frac{\partial g_{i l}}{\partial t}, \\
& \widehat{R}_{0 k 0}^{l}=\frac{1}{2} \frac{\partial g^{l i}}{\partial t} \frac{\partial g_{i k}}{\partial t}+\frac{1}{2} g^{l i} \frac{\partial^{2} g_{i k}}{\partial t^{2}}+\frac{1}{4} g^{l m} g^{p n} \frac{\partial g_{m p}}{\partial t} \frac{\partial g_{n k}}{\partial t} .
\end{aligned}
$$


Then,

$$
\begin{aligned}
\widehat{R}_{i k}= & g^{\alpha \beta} \widehat{R}_{i \alpha k \beta}=g^{\alpha \beta} g_{k l} \widehat{R}_{i \alpha \beta}^{l}=-g_{k l} \widehat{R}_{i 00}^{l}+g^{p q} g_{k l} \widehat{R}_{i p q}^{l} \\
= & -g_{k l}\left[-\frac{1}{2} \frac{\partial g^{l p}}{\partial t} \frac{\partial g_{i p}}{\partial t}-\frac{1}{2} g^{l p} \frac{\partial^{2} g_{i p}}{\partial t^{2}}-\frac{1}{4} g^{l m} g^{p n} \frac{\partial g_{m p}}{\partial t} \frac{\partial g_{n i}}{\partial t}\right] \\
& +g^{p q} g_{k l}\left[R_{i p q}^{l}+\frac{1}{4} g^{l m} \frac{\partial g_{m i}}{\partial t} \frac{\partial g_{p q}}{\partial t}-\frac{1}{4} g^{l m} \frac{\partial g_{m p}}{\partial t} \frac{\partial g_{i q}}{\partial t}\right] \\
= & \frac{1}{2} \frac{\partial^{2} g_{i k}}{\partial t^{2}}+R_{i k}+\frac{1}{2} g_{k l} \frac{\partial g^{l p}}{\partial t} \frac{\partial g_{i p}}{\partial t}+\frac{1}{4} g^{p n} \frac{\partial g_{k p}}{\partial t} \frac{\partial g_{n i}}{\partial t} \\
+ & \frac{1}{4} g^{p q} \frac{\partial g_{k i}}{\partial t} \frac{\partial g_{p q}}{\partial t}-\frac{1}{4} g^{p q} \frac{\partial g_{k p}}{\partial t} \frac{\partial g_{i q}}{\partial t} \\
= & \frac{1}{2} \frac{\partial^{2} g_{i k}}{\partial t^{2}}+R_{i k}+\frac{1}{4} g^{p q} \frac{\partial g_{i k}}{\partial t} \frac{\partial g_{p q}}{\partial t}-\frac{1}{2} g^{p q} \frac{\partial g_{i p}}{\partial t} \frac{\partial g_{k q}}{\partial t} .
\end{aligned}
$$

A direct computation gives (see, e.g., Fock [4], p.423; Fisher and Marsden [3], p.22)

$$
\widehat{R}_{i j}=\widehat{R}_{i j}^{(h)}+\frac{1}{2}\left(g_{i \alpha} \frac{\partial \widehat{\Gamma}^{\alpha}}{\partial x^{j}}+g_{j \alpha} \frac{\partial \widehat{\Gamma}^{\alpha}}{\partial x^{i}}\right)=\widehat{R}_{i j}^{(h)}+\frac{1}{2}\left(g_{i k} \frac{\partial \Gamma^{k}}{\partial x^{j}}+g_{j k} \frac{\partial \Gamma^{k}}{\partial x^{i}}\right),
$$

where

$$
\begin{gathered}
\widehat{\Gamma}^{\alpha}=g^{\beta \gamma} \widehat{\Gamma}_{\beta \gamma}^{\alpha} \text {, i.e., } \widehat{\Gamma}^{0}=g^{k l} \widehat{\Gamma}_{k l}^{0}=\frac{1}{2} g^{k l} \frac{\partial g_{k l}}{\partial t}, \widehat{\Gamma}^{i}=g^{k l} \widehat{\Gamma}_{k l}^{i}=g^{k l} \Gamma_{k l}^{i} \triangleq \Gamma^{i}, \\
\widehat{R}_{i j}^{(h)}=-\frac{1}{2} g^{\alpha \beta} \frac{\partial^{2} g_{i j}}{\partial x^{\alpha} \partial x^{\beta}}+\widehat{H}_{i j}\left(g_{\alpha \beta}, \frac{\partial g_{\alpha \beta}}{\partial x^{\lambda}}\right) \\
=\frac{1}{2} \frac{\partial^{2} g_{i j}}{\partial t^{2}}-\frac{1}{2} g^{k l} \frac{\partial^{2} g_{i j}}{\partial x^{k} \partial x^{l}}+\widehat{H}_{i j}\left(g_{\alpha \beta}, \frac{\partial g_{\alpha \beta}}{\partial x^{\lambda}}\right)
\end{gathered}
$$

and

$$
\begin{aligned}
\widehat{H}_{i j}\left(g_{\alpha \beta}, \frac{\partial g_{\alpha \beta}}{\partial x^{\lambda}}\right) & =g^{\alpha \beta} g_{e \sigma} \widehat{\Gamma}_{i \beta}^{e} \widehat{\Gamma}_{j \alpha}^{\sigma} \\
& +\frac{1}{2}\left(\frac{\partial g_{i j}}{\partial x^{\alpha}} \widehat{\Gamma}^{\alpha}+g_{j \lambda} \widehat{\Gamma}_{\alpha \beta}^{\lambda} g^{\alpha \gamma} g^{\beta g} \frac{\partial g_{\gamma g}}{\partial x^{i}}+g_{i \lambda} \widehat{\Gamma}_{\alpha \beta}^{\lambda} g^{\alpha \gamma} g^{\beta g} \frac{\partial g_{\gamma g}}{\partial x^{j}}\right) .
\end{aligned}
$$

Similar to (3.3), we have

$$
\widehat{R}_{i j}^{(h)}=\frac{1}{2} \frac{\partial^{2} g_{i j}}{\partial t^{2}}-\frac{1}{2} g^{k l} \frac{\partial^{2} g_{i j}}{\partial x^{k} \partial x^{l}}+H_{i j}\left(g_{k l}, \frac{\partial g_{k l}}{\partial x^{p}}, \frac{\partial g_{k l}}{\partial t}\right)
$$


and

$$
\begin{aligned}
H_{i j} & \left(g_{k l}, \frac{\partial g_{k l}}{\partial x^{p}}, \frac{\partial g_{k l}}{\partial t}\right) \triangleq \widehat{H}_{i j}\left(g_{\alpha \beta}, \frac{\partial g_{\alpha \beta}}{\partial x^{\lambda}}\right) \\
= & -g_{k l} \widehat{\Gamma}_{i 0}^{k} \widehat{\Gamma}_{j 0}^{l}+(-1) g^{k l} \widehat{\Gamma}_{i k}^{0} \widehat{\Gamma}_{j l}^{0}+g^{k l} g_{p q} \Gamma_{i k}^{p} \Gamma_{j l}^{q} \\
& +\frac{1}{2}\left(\frac{\partial g_{i j}}{\partial t} \frac{1}{2} g^{k l} \frac{\partial g_{k l}}{\partial t}+\frac{\partial g_{i j}}{\partial x_{k}} \Gamma_{p q}^{k} g^{p q}+g_{j k} \Gamma_{r s}^{k} \frac{\partial g_{p q}}{\partial x^{i}} g^{p r} g^{q s}+g_{i k} \Gamma_{r s}^{k} g^{p r} g^{q s} \frac{\partial g_{p q}}{\partial x^{j}}\right) \\
= & -g_{k l} \frac{1}{2} g^{k p} \frac{\partial g_{i p}}{\partial t} \frac{1}{2} g^{l q} \frac{\partial g_{j q}}{\partial t}-g^{k l} \frac{1}{2} \frac{\partial g_{i k}}{\partial t} \frac{1}{2} \frac{\partial g_{j l}}{\partial t}+g^{k l} g_{p q} \Gamma_{i k}^{p} \Gamma_{j l}^{q} \\
& +\frac{1}{4} g^{k l} \frac{\partial g_{k l}}{\partial t} \frac{\partial g_{i j}}{\partial t}+\frac{1}{2} \frac{\partial g_{i j}}{\partial x_{k}} \Gamma_{p q}^{k} g^{p q} \\
& +\frac{1}{2}\left(g_{i k} \Gamma_{r s}^{k} g^{p r} g^{q s} \frac{\partial g_{p q}}{\partial x^{j}}+g_{j k} \Gamma_{r s}^{k} g^{p r} g^{q s} \frac{\partial g_{p q}}{\partial x^{i}}\right) \\
= & -\frac{1}{2} g^{p q} \frac{\partial g_{i p}}{\partial t} \frac{\partial g_{j q}}{\partial t}+\frac{1}{4} g^{k l} \frac{\partial g_{k l}}{\partial t} \frac{\partial g_{i j}}{\partial t} \\
& +g^{k l} g_{p q} \Gamma_{i k}^{p} \Gamma_{j l}^{q}+\frac{1}{2} \frac{\partial g_{i j}}{\partial x_{k}} \Gamma_{p q}^{k} g^{p q} \\
& +\frac{1}{2}\left(g_{i k} \Gamma_{r s}^{k} g^{p r} g^{q s} \frac{\partial g_{p q}}{\partial x^{j}}+g_{j k} \Gamma_{r s}^{k} g^{p r} g^{q s} \frac{\partial g_{p q}}{\partial x^{i}}\right) .
\end{aligned}
$$

It follows from (3.3) that

$$
\begin{aligned}
\frac{1}{2} \frac{\partial^{2} g_{i j}}{\partial t^{2}}+R_{i j}= & \frac{1}{2} \frac{\partial^{2} g_{i j}}{\partial t^{2}}-\frac{1}{2} g^{k l} \frac{\partial^{2} g_{i j}}{\partial x^{k} \partial x^{l}}+\frac{1}{2}\left(g_{i k} \frac{\partial \Gamma^{k}}{\partial x^{j}}+g_{j k} \frac{\partial \Gamma^{k}}{\partial x^{i}}\right) \\
& +H_{i j}\left(g_{k l}, \frac{\partial g_{k l}}{\partial x^{p}}, \frac{\partial g_{k l}}{\partial t}\right)+\frac{1}{2} g^{p q} \frac{\partial g_{i p}}{\partial t} \frac{\partial g_{j q}}{\partial t}-\frac{1}{4} g^{p q} \frac{\partial g_{i j}}{\partial t} \frac{\partial g_{p q}}{\partial t}
\end{aligned}
$$

i.e.,

$$
\begin{aligned}
\frac{\partial^{2} g_{i j}}{\partial t^{2}}+2 R_{i j}= & \frac{\partial^{2} g_{i j}}{\partial t^{2}}-g^{k l} \frac{\partial^{2} g_{i j}}{\partial x^{k} \partial x^{l}}+\left(g_{i k} \frac{\partial \Gamma^{k}}{\partial x^{j}}+g_{j k} \frac{\partial \Gamma^{k}}{\partial x^{i}}\right) \\
& +2 H_{i j}\left(g_{k l}, \frac{\partial g_{k l}}{\partial x^{p}}, \frac{\partial g_{k l}}{\partial t}\right)+g^{p q} \frac{\partial g_{i p}}{\partial t} \frac{\partial g_{j q}}{\partial t}-\frac{1}{2} g^{p q} \frac{\partial g_{i j}}{\partial t} \frac{\partial g_{p q}}{\partial t} \\
= & \frac{\partial^{2} g_{i j}}{\partial t^{2}}-g^{k l} \frac{\partial^{2} g_{i j}}{\partial x^{k} \partial x^{l}}+\left(g_{i k} \frac{\partial \Gamma^{k}}{\partial x^{j}}+g_{j k} \frac{\partial \Gamma^{k}}{\partial x^{i}}\right) \\
& +2 g^{k l} g_{p q} \Gamma_{i k}^{p} \Gamma_{j l}^{q}+\frac{\partial g_{i j}}{\partial x_{k}} \Gamma_{p q}^{k} g^{p q} \\
& +\left(g_{i k} \Gamma_{r s}^{k} g^{p r} g^{q s} \frac{\partial g_{p q}}{\partial x^{j}}+g_{j k} \Gamma_{r s}^{k} g^{p r} g^{q s} \frac{\partial g_{p q}}{\partial x^{i}}\right)
\end{aligned}
$$


Similar to the harmonic coordinates in the space-time (see [3]), here we make use of a new kind of coordinates on the manifold $\mathscr{M}$ defined by

$$
\Gamma^{i} \triangleq g^{k l} \Gamma_{k l}^{i}=0
$$

Such new coordinates are called elliptic coordinates on $\mathscr{M}$.

Lemma 3.1. Let $g_{i j}$ be a $C^{\infty}$ Riemannian metric on the manifold $\mathscr{M}$. There is a $C^{\infty}$ local coordinates transformation $\phi: \mathscr{M} \longrightarrow \mathscr{M}, x \longrightarrow \bar{x}$ around a fixed point $p \in \mathscr{M}$ such that the transformed metric $\bar{g}_{i j}$ is a $C^{\infty}$ Riemannian metric with $\bar{\Gamma}^{k}(\bar{x})=0$ for all $\bar{x}$ in a neighborhood around $p \in \mathscr{M}$ and any $k \in\{1,2, \cdots, n\}$.

Proof. Consider the elliptic equation for the scalar $\psi$,

$$
\triangle \psi \triangleq-g^{k l} \frac{\partial^{2} \psi}{\partial x_{k} \partial x_{l}}+g^{k l} \Gamma_{k l}^{j} \frac{\partial \psi}{\partial x_{j}}=0 .
$$

The coefficients are $C^{\infty}$. Let $\bar{x}^{i}(x)$ be a solution with the condition $\bar{x}^{i}(p)=$ $x^{i}(p), \quad \frac{\partial \bar{x}^{i}}{\partial x_{j}}(p)=\delta_{j}^{i}$. Then $\bar{x}^{i}$ is a $C^{\infty}$ local coordinates transformation $\phi:$ $\mathscr{M} \longrightarrow \mathscr{M}$ around $p$ and the transformed metric $\bar{g}_{i j}$ is a $C^{\infty}$ Riemannian metric.

Now the equation $\triangle \psi=0$ is a tensorial (scalar) equation. In the barred coordinates, it becomes

$$
0=\bar{\triangle} \bar{x}^{k}=-\bar{g}^{i j} \frac{\partial^{2} \bar{x}^{k}}{\partial \bar{x}_{i} \partial \bar{x}_{j}}+\bar{\Gamma}^{j} \frac{\partial \bar{x}^{k}}{\partial \bar{x}_{j}}=\bar{\Gamma}^{k} .
$$

Therefore, $\bar{g}_{i j}$ satisfies the elliptic condition (3.5). The proof of Lemma 3.1 is complete.

By Lemma 3.1, we can choose the elliptic coordinates around a fixed point $p \in \mathscr{M}$ and for a fixed time $t \in \mathbb{R}^{+}$. After throwing off the bar sign, the geometric hyperbolic flow (3.1) can be written as

$$
\frac{\partial^{2} g_{i j}}{\partial t^{2}}=g^{k l} \frac{\partial^{2} g_{i j}}{\partial x^{k} \partial x^{l}}+\widetilde{H}_{i j}\left(g_{k l}, \frac{\partial g_{k l}}{\partial x^{p}}\right),
$$

where

$$
\widetilde{H}_{i j}\left(g_{k l}, \frac{\partial g_{k l}}{\partial x^{p}}\right)=-2 g^{k l} g_{p q} \Gamma_{i k}^{p} \Gamma_{j l}^{q}-\left(g_{i k} \Gamma_{r s}^{k} g^{p r} g^{q s} \frac{\partial g_{p q}}{\partial x^{j}}+g_{j k} \Gamma_{r s}^{k} g^{p r} g^{q s} \frac{\partial g_{p q}}{\partial x^{i}}\right)
$$


are homogenous quadratic with respect to $\frac{\partial g_{k l}}{\partial x^{p}}$ and rational with respect to $g_{k l}$ with non-zero denominator $\operatorname{det}(\mathrm{g}) \neq 0$. By introducing the new unknowns $g_{i j}, h_{i j}=\frac{\partial g_{i j}}{\partial t}, g_{i j, k}=\frac{\partial g_{i j}}{\partial x^{k}}$, the system (3.6) can be reduced to a system of partial differential equations of first order. We now consider such a quasi-linear (symmetric hyperbolic) system with $\frac{1}{2} n(n+1)(n+2)$ PDEs of first order

$$
\left\{\begin{array}{l}
\frac{\partial g_{i j}}{\partial t}=h_{i j}, \\
g^{k l} \frac{\partial g_{i j, k}}{\partial t}=g^{k l} \frac{\partial h_{i j}}{\partial x^{k}}, \\
\frac{\partial h_{i j}}{\partial t}=g^{k l} \frac{\partial g_{i j, k}}{\partial x^{l}}+\widetilde{H}_{i j} .
\end{array}\right.
$$

In the $C^{2}$ class, the system (3.8) is equivalent to (3.6).

Let $u=\left(g_{i j}, g_{i j, k}, h_{i j}\right)^{T}$ be the $\frac{1}{2} n(n+1)(n+2)$-dimensional unknown vector function. The coefficient matrices $A^{0}, A^{j}, B$ are given by

$$
\begin{gathered}
A^{0}(u)=A^{0}\left(g_{i j}, g_{i j, k}, h_{i j}\right)= \\
\left(\begin{array}{llllll}
I & 0 & 0 & \cdots & 0 & 0 \\
0 & g^{11} I & g^{12} I & \cdots & g^{1 n} I & 0 \\
0 & g^{21} I & g^{22} I & \cdots & g^{2 n} I & 0 \\
\vdots & \cdots & & & & \\
0 & g^{n 1} I & g^{n 2} I & \cdots & g^{n n} I & 0 \\
0 & 0 & 0 & \cdots & 0 & I
\end{array}\right), \\
A^{j}(u)=A^{j}\left(g_{k l}, g_{k l, p}, h_{k l}\right)= \\
\left(\begin{array}{cccccc}
0 & 0 & 0 & \cdots & 0 & 0 \\
0 & 0 & 0 & \cdots & 0 & g^{j 1} I \\
0 & 0 & 0 & \cdots & 0 & g^{j 2} I \\
\cdots & \cdots & \cdots & & & \\
0 & 0 & 0 & \cdots & 0 & g^{j n} I \\
0 & g^{1 j} I & g^{2 j} I & \cdots & g^{n j} I & 0
\end{array}\right),
\end{gathered}
$$

where 0 is the $\left(\frac{1}{2} n(n+1)\right) \times\left(\frac{1}{2} n(n+1)\right)$ zero matrix, $I$ is the $\left(\frac{1}{2} n(n+1)\right) \times$ $\left(\frac{1}{2} n(n+1)\right)$ identity matrix

$$
B(u)=B\left(g_{i j}, g_{i j, p}, h_{i j}\right)=\left(\begin{array}{c}
h_{i j} \\
0 \\
\widetilde{H}_{i j}
\end{array}\right),
$$


in which 0 is the $\frac{1}{2} n^{2}(n+1)$-dimensional zero vector.

We observe that the symmetric hyperbolic system

$$
A^{0}(u) \frac{\partial u}{\partial t}=A^{j}(u) \frac{\partial u}{\partial x^{j}}+B(u)
$$

is nothing but the system (3.8). So far, we have reduced the equation of the hyperbolic geometric flow (3.1) to the symmetric hyperbolic system (3.9), which are equivalent to each other in the $C^{2}$ class. Then, by the theory of the symmetric hyperbolic system, the smooth solution to (3.1) exists uniquely for a short time (see [3]). Thus, the proof of Theorem 1.1 is completed.

Remark 3.1. The elliptic coordinates can also be used to prove the short-time existence for the Ricci flow.

More generally, motivated by general Einstein equations and the rich theory of hyperbolic equations, we may also consider the following field equations with the energy-momentum tensor $T_{i j}$ under certain conditions:

$$
\alpha_{i j} \frac{\partial^{2} g_{i j}}{\partial t^{2}}+2 R_{i j}+\mathscr{F}_{i j}\left(g, \frac{\partial g}{\partial t}\right)=\kappa T_{i j},
$$

where $\alpha_{i j}$ are certain smooth functions on $\mathscr{M}$ which may also depend on $t, \mathscr{F}_{i j}$ are some given smooth functions of the Riemannian metric $g$ and its first order derivative with respect to $t$, and $\kappa$ is a parameter. Similar results can be obtained.

Remark 3.2. For noncompact manifolds, if the initial metric, velocity and curvature satisfy some bounded conditions, then a theory similar to Shi's short-time existence result can be developed. That is to say, under suitable assumptions, Shi's short-time existence result for the ricci flow can be extended to the hyperbolic geometric flow.

\section{NONLINEAR STABILITy FOR HYPERBOLIC GEOMETRIC FLOW}

In this section we investigate the nonlinear stability of the hyperbolic geometric flow defined on the Euclidean space with the dimension larger than 4 .

We now state the definition of nonlinear stability of the hyperbolic geometric flow (1.1). Let $\mathscr{M}$ be an $n$-dimensional complete Riemannian manifold. Given 
symmetric tensors $g_{i j}^{0}$ and $g_{i j}^{1}$ on $\mathscr{M}$, we consider the following initial value problem

$$
\left\{\begin{array}{l}
\frac{\partial^{2}}{\partial t^{2}} g_{i j}(x, t)=-2 R_{i j}(x, t), \\
g_{i j}(x, 0)=\bar{g}_{i j}(x)+\varepsilon g_{i j}^{0}(x), \quad \frac{\partial g_{i j}}{\partial t}(x, 0)=\varepsilon g_{i j}^{1}(x),
\end{array}\right.
$$

where $\varepsilon>0$ is a small parameter.

Definition 4.1. The Ricci flat Riemannian metric $\bar{g}_{i j}(x)$ possesses the (locally) nonlinear stability with respect to $\left(g_{i j}^{0}, g_{i j}^{1}\right)$, if there exists a positive constant $\varepsilon_{0}=$ $\varepsilon_{0}\left(g_{i j}^{0}, g_{i j}^{1}\right)$ such that, for any $\varepsilon \in\left(0, \varepsilon_{0}\right]$, the initial value problem (4.1) has a unique (local) smooth solution $g_{i j}(x, t)$;

$\bar{g}_{i j}(x)$ is said to be (locally) nonlinearly stable, if it possesses the (locally) nonlinear stability with respect to arbitrary symmetric tensors $g_{i j}^{0}(x)$ and $g_{i j}^{1}(x)$ with compact support.

In what follows, we consider the nonlinear stability of the flat metric of the Euclidean space $\mathbb{R}^{n}$ with the dimension $n \geq 5$. We have

Theorem 4.1. The flat metric $g_{i j}=\delta_{i j}$ of the Euclidean space $\mathbb{R}^{n}$ with $n \geq 5$ is nonlinearly stable.

Remark 4.1. Theorem 4.1 gives the nonlinear stability of the hyperbolic geometric flow on the Euclidean space with dimension larger than 4. The situation for the 3-, 4-dimensional Euclidean spaces is very different, and will be studied in the sequel by using null conditions. This is similar to the proofs of the Poincaré conjecture in topology: the proofs for the three and four dimensional case and $n \geq 5$ dimensional case are very different (see, for example, [6] and [1]). This motivates us to understand the possibility of using hyperbolic geometric flow to understand Poincaré conjecture.

Proof of Theorem 4.1. Define a 2-tensor $h$ in the following way

$$
g_{i j}(x, t)=\delta_{i j}+h_{i j}(x, t) .
$$

Let $\delta^{i j}$ be the inverse of $\delta_{i j}$. Then, for small $h$

$$
H^{i j} \triangleq g^{i j}-\delta^{i j}=-h^{i j}+O^{i j}\left(h^{2}\right),
$$

where $h^{i j}=\delta^{i k} \delta^{j l} h_{k l}$ and $O^{i j}\left(h^{2}\right)$ vanishes to second order at $h=0$. 
As in Section 3, we choose the above elliptic coordinates $\left\{x^{i}\right\}$ around the origin in $\mathbb{R}^{n}$. Then the initial value problem (4.1) can be written as

$$
\left\{\begin{array}{l}
\frac{\partial^{2}}{\partial t^{2}} h_{i j}(x, t)=\left(\delta^{k l}+H^{k l}\right) \frac{\partial^{2} h_{i j}}{\partial x^{k} \partial x^{l}}+\tilde{H}_{i j}\left(\delta_{k l}+h_{k l}(x, t), \frac{\partial h_{k l}(x, t)}{\partial x^{p}}\right), \\
h_{i j}(x, 0)=\varepsilon g_{i j}^{0}(x), \quad \frac{\partial}{\partial t} h_{i j}(x, 0)=\varepsilon g_{i j}^{1}(x),
\end{array}\right.
$$

where

$$
\begin{aligned}
& \tilde{H}_{i j}\left(\delta_{k l}+h_{k l}(x, t), \frac{\partial h_{k l}(x, t)}{\partial x^{p}}\right)=\tilde{H}_{i j}\left(g_{k l}(x, t), \frac{\partial g_{k l}(x, t)}{\partial x^{p}}\right) \\
=- & {\left[2 g^{k l} g_{p q} \Gamma_{i k}^{p} \Gamma_{j l}^{q}+g_{i k} \Gamma_{r s}^{k} g^{p r} g^{q s} \frac{\partial g_{p q}}{\partial x^{j}}+g_{j k} \Gamma_{r s}^{k} g^{p r} g^{q s} \frac{\partial g_{p q}}{\partial x^{i}}\right] } \\
= & -2\left(\delta^{k l}+H^{i j}\right)\left(\delta_{p q}+h_{p q}\right) \frac{1}{4}\left(\delta^{p a}+H^{p a}\right)\left(\delta^{q b}+H^{q b}\right) \\
& \cdot\left(\frac{\partial h_{a i}}{\partial x^{k}}+\frac{\partial h_{a k}}{\partial x^{i}}-\frac{\partial h_{i k}}{\partial x^{a}}\right)\left(\frac{\partial h_{b j}}{\partial x^{l}}+\frac{\partial h_{b l}}{\partial x^{j}}-\frac{\partial h_{j l}}{\partial x^{b}}\right) \\
- & \left(\delta_{i k}+H_{i k}\right)\left(\delta^{p r}+H^{p r}\right)\left(\delta^{q s}+H^{q s}\right) \\
& \cdot \frac{1}{2}\left(\delta^{k a}+H^{k a}\right)\left(\frac{\partial h_{a r}}{\partial x^{s}}+\frac{\partial h_{a s}}{\partial x^{r}}-\frac{\partial h_{r s}}{\partial x^{a}}\right) \frac{\partial h_{p q}}{\partial x^{j}} \\
- & \left(\delta_{j k}+H_{j k}\right)\left(\delta^{p r}+H^{p r}\right)\left(\delta^{q s}+H^{q s}\right) \\
& \cdot \frac{1}{2}\left(\delta^{k a}+H^{k a}\right)\left(\frac{\partial h_{a r}}{\partial x^{s}}+\frac{\partial h_{a s}}{\partial x^{r}}-\frac{\partial h_{r s}}{\partial x^{a}}\right) \frac{\partial h_{p q}}{\partial x^{i}} \\
=- & \cdot \frac{1}{4} \cdot \delta^{k l} \delta_{p q} \delta^{p a} \delta^{q b}\left(\frac{\partial h_{a i}}{\partial x^{k}}+\frac{\partial h_{a k}}{\partial x^{i}}-\frac{\partial h_{i k}}{\partial x^{a}}\right)\left(\frac{\partial h_{b j}}{\partial x^{l}}+\frac{\partial h_{b l}}{\partial x^{j}}-\frac{\partial h_{j l}}{\partial x^{b}}\right) \\
- & \frac{1}{2} \delta_{i k} \delta^{p r} \delta^{q s} \delta^{k a}\left(\frac{\partial h_{a r}}{\partial x^{s}}+\frac{\partial h_{a s}}{\partial x^{r}}-\frac{\partial h_{r s}}{\partial x^{a}}\right) \frac{\partial h_{p q}}{\partial x^{j}} \\
- & \frac{1}{2} \delta_{j k} \delta^{p r} \delta^{q s} \delta^{k a}\left(\frac{\partial h_{a r}}{\partial x^{s}}+\frac{\partial h_{a s}}{\partial x^{r}}-\frac{\partial h_{r s}}{\partial x^{a}}\right) \frac{\partial h_{p q}}{\partial x^{i}}+O\left(\left|h_{k l}\right|+\left|\frac{\partial h_{k l}}{\partial x^{p}}\right|\right)^{3} \\
=- & \frac{1}{2} \delta^{k l} \delta^{a b}\left(\frac{\partial h_{a i}}{\partial x^{k}}+\frac{\partial h_{a k}}{\partial x^{i}}-\frac{\partial h_{i k}}{\partial x^{a}}\right)\left(\frac{\partial h_{b j}}{\partial x^{l}}+\frac{\partial h_{b l}}{\partial x^{j}}-\frac{\partial h_{j l}}{\partial x^{b}}\right) \\
- & \frac{1}{2} \delta^{p r} \delta^{q s}\left(\frac{\partial h_{i r}}{\partial x^{s}}+\frac{\partial h_{i s}}{\partial x^{r}}-\frac{\partial h_{r s}}{\partial x^{i}}\right) \frac{\partial h_{p q}}{\partial x^{j}} \\
- & \frac{\partial}{\partial \delta^{p r}}\left(\frac{\partial h_{j r}}{\partial x^{s}}+\frac{\partial h_{j s}}{\partial x^{r}}-\frac{\partial h_{r s}}{\partial x^{j}}\right) \frac{\partial h_{p q}}{\partial x^{i}}+O\left(\left|h_{k l}\right|+\left|\frac{\partial h_{k l}}{\partial x^{p}}\right|\right)^{3} \cdot \\
& \\
& \\
&
\end{aligned}
$$


Furthermore, (4.2) can be written as

$$
\left\{\begin{array}{l}
\frac{\partial^{2}}{\partial t^{2}} h_{i j}(x, t)=\sum_{k=1}^{n} \frac{\partial^{2} h_{i j}}{\partial x^{k} \partial x^{k}}+\bar{H}_{i j}\left(h_{k l}, \frac{\partial h_{k l}}{\partial x^{p}}, \frac{\partial^{2} h_{k l}}{\partial x^{p} \partial x^{q}}\right), \\
h_{i j}(x, 0)=\varepsilon g_{i j}^{0}(x), \quad \frac{\partial}{\partial t} h_{i j}(x, 0)=\varepsilon g_{i j}^{1}(x),
\end{array}\right.
$$

where

$$
\begin{gathered}
\bar{H}_{i j}\left(h_{k l}, \frac{\partial h_{k l}}{\partial x^{p}}, \frac{\partial^{2} h_{k l}}{\partial x^{p} \partial x^{q}}\right)=H^{k l} \frac{\partial^{2} h_{i j}}{\partial x^{k} x^{l}}+\widetilde{H}_{i j}\left(\delta_{k l}+h_{k l}, \frac{\partial h_{k l}}{\partial x^{p}}\right) \\
=-\frac{1}{2} \delta^{k l} \delta^{a b}\left(\frac{\partial h_{a i}}{\partial x^{k}}+\frac{\partial h_{a k}}{\partial x^{i}}-\frac{\partial h_{i k}}{\partial x^{a}}\right)\left(\frac{\partial h_{b j}}{\partial x^{l}}+\frac{\partial h_{b l}}{\partial x^{j}}-\frac{\partial h_{j l}}{\partial x^{b}}\right) \\
-\frac{1}{2} \delta^{p r} \delta^{q s}\left(\frac{\partial h_{i r}}{\partial x^{s}}+\frac{\partial h_{i s}}{\partial x^{r}}-\frac{\partial h_{r s}}{\partial x^{i}}\right) \frac{\partial h_{p q}}{\partial x^{j}} \\
-\frac{1}{2} \delta^{p r} \delta^{q s}\left(\frac{\partial h_{j r}}{\partial x^{s}}+\frac{\partial h_{j s}}{\partial x^{r}}-\frac{\partial h_{r s}}{\partial x^{j}}\right) \frac{\partial h_{p q}}{\partial x^{i}} \\
-h^{k l} \frac{\partial^{2} h_{i j}}{\partial x^{k} \partial x^{l}}+O\left(\left|h_{k l}\right|+\left|\frac{\partial h_{k l} \mid}{\partial x^{p}}\right|+\left|\frac{\partial^{2} h_{k l}}{\partial x^{p} \partial x^{q}}\right|\right)^{3} .
\end{gathered}
$$

Let

$$
\hat{\lambda}=\left(h_{k l}, \frac{\partial h_{k l}}{\partial x^{p}}, \frac{\partial^{2} h_{k l}}{\partial x^{p} \partial x^{q}}\right) \quad(p, q, k, l=1,2 \cdots, n) .
$$

The nonlinear term

$$
\bar{H}_{i j}(\hat{\lambda})=\bar{H}_{i j}\left(h_{k l}, \frac{\partial h_{k l}}{\partial x^{p}}, \frac{\partial^{2} h_{k l}}{\partial x^{p} \partial x^{q}}\right)
$$

in (4.3) is smooth in a neighborhood about $\hat{\lambda}=0$ and satisfies

$$
\bar{H}_{i j}(\hat{\lambda})=O\left(|\hat{\lambda}|^{2}\right) \quad(i, j=1,2, \cdots, n) .
$$

By the well-known global existence results for the nonlinear wave equation (e.g., see [2], [7], [8], [10]), there exists a unique global smooth solution $\left(h_{i j}(x, t)\right)$ for the Cauchy problem (4.3) or (4.2). Thus, the proof of Theorem 4.1 is complete.

Remark 4.2. For general complete noncompact Riemannian manifold $\mathscr{M}$, under suitable bounded assumptions we can prove that any Ricci flat Riemannian metric $\bar{g}_{i j}(x)$ on $\mathscr{M}$ possesses the locally nonlinear stability. For the globally nonlinear stability, when the space dimension $n$ is large that 4 , i.e., $n \geq 5$, under suitable assumptions, we can show that the Ricci flat Riemannian metric $\bar{g}_{i j}(x)$ on 
$\mathscr{M}$ possesses the globally nonlinear stability, however when $n=3,4$, this result in general is not true, since in this situation the solution to the corresponding nonlinear wave equation in general blows up in finite time.

\section{WAVE CHARACTER OF THE CURVATURES}

The hyperbolic geometric flow is a system of hyperbolic evolution equations on the metrics. The evolution of the metrics implies a system of nonlinear wave equations for the Riemannian curvature tensor $R_{i j k l}$, the Ricci curvature tensor $R_{i j}$ and the scalar curvature $R$ which we will derive.

Let $\mathscr{M}$ be an $n$-dimensional complete manifold. We consider the hyperbolic geometric flow on $\mathscr{M}$, that is,

$$
\frac{\partial^{2}}{\partial t^{2}} g_{i j}(x, t)=-2 R_{i j}(x, t)
$$

We now want to find the evolution equations for the Riemannian curvature tensor $R_{i j k l}$, the Ricci curvature tensor $R_{i j}$ and the scalar curvature $R$.

Direct computations yield

$$
\begin{aligned}
\Gamma_{j l}^{h}= & \frac{1}{2} g^{h m}\left(\frac{\partial g_{m j}}{\partial x^{l}}+\frac{\partial g_{m l}}{\partial x^{j}}-\frac{\partial g_{j l}}{\partial x^{m}}\right), \\
\frac{\partial}{\partial t} \Gamma_{j l}^{h}= & \frac{1}{2} g^{h m}\left(\frac{\partial^{2} g_{m j}}{\partial x^{l} \partial t}+\frac{\partial^{2} g_{m l}}{\partial x^{j} \partial t}-\frac{\partial^{2} g_{j l}}{\partial x^{m} \partial t}\right)+\frac{1}{2} \frac{\partial g^{h m}}{\partial t}\left(\frac{\partial g_{m j}}{\partial x^{l}}+\frac{\partial g_{m l}}{\partial x^{j}}-\frac{\partial g_{j l}}{\partial x^{m}}\right), \\
\frac{\partial^{2}}{\partial t^{2}} \Gamma_{j l}^{h}= & \frac{1}{2} \frac{\partial^{2} g^{h m}}{\partial t^{2}}\left(\frac{\partial g_{m j}}{\partial x^{l}}+\frac{\partial g_{m l}}{\partial x^{j}}-\frac{\partial g_{j l}}{\partial x^{m}}\right)+2 \cdot \frac{1}{2} \frac{\partial g^{h m}}{\partial t}\left(\frac{\partial^{2} g_{m j}}{\partial x^{l} \partial t}+\frac{\partial^{2} g_{m l}}{\partial x^{j} \partial t}-\frac{\partial^{2} g_{j l}}{\partial x^{m} \partial t}\right) \\
& +\frac{1}{2} g^{h m}\left(\frac{\partial}{\partial x^{l}}\left(\frac{\partial^{2} g_{m j}}{\partial t^{2}}\right)+\frac{\partial}{\partial x^{j}}\left(\frac{\partial^{2} g_{m l}}{\partial t^{2}}\right)-\frac{\partial}{\partial x^{m}}\left(\frac{\partial^{2} g_{j l}}{\partial t^{2}}\right)\right), \\
\frac{\partial^{2}}{\partial t^{2}} R_{i j l}^{h}= & \frac{\partial}{\partial x^{i}}\left(\frac{\partial^{2}}{\partial t^{2}} \Gamma_{j l}^{h}\right)-\frac{\partial}{\partial x^{j}}\left(\frac{\partial^{2}}{\partial t^{2}} \Gamma_{i l}^{h}\right)+\frac{\partial^{2}}{\partial t^{2}}\left(\Gamma_{i p}^{h} \Gamma_{j l}^{p}-\Gamma_{j p}^{h} \Gamma_{i l}^{p}\right), \\
\frac{\partial^{2}}{\partial t^{2}} R_{i j k l}= & \frac{\partial^{2}}{\partial t^{2}}\left(g_{h k} R_{i j l}^{h}\right)=g_{h k} \frac{\partial^{2}}{\partial t^{2}} R_{i j l}^{h}+R_{i j l}^{h} \frac{\partial^{2} g_{h k}}{\partial t^{2}}+2 \frac{\partial g_{h k}}{\partial t} \frac{\partial}{\partial t} R_{i j l}^{h} \\
& =g_{h k}\left[\frac{\partial}{\partial x^{i}}\left(\frac{\partial^{2}}{\partial t^{2}} \Gamma_{j l}^{h}\right)-\frac{\partial}{\partial x^{j}}\left(\frac{\partial^{2}}{\partial t^{2}} \Gamma_{i l}^{h}\right)+\frac{\partial^{2}}{\partial t^{2}}\left(\Gamma_{i p}^{h} \Gamma_{j l}^{p}-\Gamma_{j p}^{h} \Gamma_{i l}^{p}\right)\right] \\
& +2 \frac{\partial g_{h k}}{\partial t}\left[\frac{\partial}{\partial x^{i}}\left(\frac{\partial}{\partial t} \Gamma_{j l}^{h}\right)-\frac{\partial}{\partial x^{j}}\left(\frac{\partial}{\partial t} \Gamma_{i l}^{h}\right)+\frac{\partial}{\partial t}\left(\Gamma_{i p}^{h} \Gamma_{j l}^{p}-\Gamma_{j p}^{h} \Gamma_{i l}^{p}\right)\right] \\
& +R_{i j l}^{h} \frac{\partial^{2} g_{h k}}{\partial t^{2}} .
\end{aligned}
$$


We choose the normal coordinates around a fixed point $p$ on $\mathscr{M}$ such that

$$
\Gamma_{i j}^{k}(p)=0,
$$

or, equivalently,

$$
\frac{\partial g_{i j}}{\partial x^{k}}(p)=0 .
$$

Then we have

$$
\begin{aligned}
& \frac{\partial^{2}}{\partial t^{2}} R_{i j k l}= \\
& g_{h k} \frac{\partial}{\partial x^{i}}\left[\frac{1}{2} \frac{\partial^{2} g^{h m}}{\partial t^{2}}\left(\frac{\partial g_{m j}}{\partial x^{l}}+\frac{\partial g_{m l}}{\partial x^{j}}-\frac{\partial g_{j l}}{\partial x^{m}}\right)+2 \cdot \frac{1}{2} \frac{\partial g^{h m}}{\partial t}\left(\frac{\partial^{2} g_{m j}}{\partial x^{l} \partial t}+\frac{\partial^{2} g_{m l}}{\partial x^{j} \partial t}-\frac{\partial^{2} g_{j l}}{\partial x^{m} \partial t}\right)\right] \\
& +g_{h k} \frac{\partial}{\partial x^{i}}\left[\frac{1}{2} g^{h m}\left(\frac{\partial}{\partial x^{l}}\left(\frac{\partial^{2} g_{m j}}{\partial t^{2}}\right)+\frac{\partial}{\partial x^{j}}\left(\frac{\partial^{2} g_{m l}}{\partial t^{2}}\right)-\frac{\partial}{\partial x^{m}}\left(\frac{\partial^{2} g_{j l}}{\partial t^{2}}\right)\right)\right] \\
& -g_{h k} \frac{\partial}{\partial x^{j}}\left[\frac{1}{2} \frac{\partial g^{h m}}{\partial t^{2}}\left(\frac{\partial g_{m i}}{\partial x^{l}}+\frac{\partial g_{m l}}{\partial x^{i}}-\frac{\partial g_{i l}}{\partial x^{m}}\right)+2 \cdot \frac{1}{2} \frac{\partial g^{h m}}{\partial t}\left(\frac{\partial^{2} g_{m i}}{\partial x^{l} \partial t}+\frac{\partial^{2} g_{m l}}{\partial x^{i} \partial t}-\frac{\partial^{2} g_{i l}}{\partial x^{m} \partial t}\right)\right] \\
& -g_{h k} \frac{\partial}{\partial x^{j}}\left[\frac{1}{2} g^{h m}\left(\frac{\partial}{\partial x^{l}}\left(\frac{\partial^{2} g_{m i}}{\partial t^{2}}\right)+\frac{\partial}{\partial x^{i}}\left(\frac{\partial^{2} g_{m l}}{\partial t^{2}}\right)-\frac{\partial}{\partial x^{m}}\left(\frac{\partial^{2} g_{i l}}{\partial t^{2}}\right)\right)\right] \\
& +2 g_{h k}\left(\frac{\partial}{\partial t} \Gamma_{i p}^{h} \frac{\partial}{\partial t} \Gamma_{j l}^{p}-\frac{\partial}{\partial t} \Gamma_{j p}^{h} \frac{\partial}{\partial t} \Gamma_{i l}^{p}\right)+2 \frac{\partial g_{h k}}{\partial t} \\
& \text {. }\left[\frac{1}{2} \frac{\partial}{\partial x^{i}}\left(\frac{\partial g^{h m}}{\partial t}\left(\frac{\partial g_{m j}}{\partial x^{l}}+\frac{\partial g_{m l}}{\partial x^{j}}-\frac{\partial g_{j l}}{\partial x^{m}}\right)\right)-\frac{1}{2} \frac{\partial}{\partial x^{j}}\left(\frac{\partial g^{h m}}{\partial t}\left(\frac{\partial g_{m i}}{\partial x^{l}}+\frac{\partial g_{m l}}{\partial x^{i}}-\frac{\partial g_{i l}}{\partial x^{m}}\right)\right)\right] \\
& +2 \frac{\partial g_{h k}}{\partial t} \frac{1}{2} g^{h m} \frac{\partial}{\partial x^{i}}\left(\frac{\partial}{\partial x^{l}}\left(\frac{\partial g_{m j}}{\partial t}\right)+\frac{\partial}{\partial x^{j}}\left(\frac{\partial g_{m l}}{\partial t}\right)-\frac{\partial}{\partial x^{m}}\left(\frac{\partial g_{j l}}{\partial t}\right)\right) \\
& -2 \frac{\partial g_{h k}}{\partial t} \frac{1}{2} g^{h m} \frac{\partial}{\partial x^{j}}\left(\frac{\partial}{\partial x^{l}}\left(\frac{\partial g_{m i}}{\partial t}\right)+\frac{\partial}{\partial x^{i}}\left(\frac{\partial g_{m l}}{\partial t}\right)-\frac{\partial}{\partial x^{m}}\left(\frac{\partial g_{i l}}{\partial t}\right)\right)+R_{i j l}^{h} \frac{\partial^{2} g_{h k}}{\partial t^{2}} \\
& =\frac{1}{2} g_{h k} \frac{\partial^{2} g^{h m}}{\partial t^{2}}\left[\frac{\partial}{\partial x^{i}}\left(\frac{\partial g_{m j}}{\partial x^{l}}+\frac{\partial g_{m l}}{\partial x^{j}}-\frac{\partial g_{j l}}{\partial x^{m}}\right)-\frac{\partial}{\partial x^{j}}\left(\frac{\partial g^{h m}}{\partial t}\left(\frac{\partial g_{m i}}{\partial x^{l}}+\frac{\partial g_{m l}}{\partial x^{i}}-\frac{\partial g_{i l}}{\partial x^{m}}\right)\right)\right] \\
& +g_{h k} \frac{\partial^{2} g^{h m}}{\partial x^{i} \partial t}\left(\frac{\partial g_{m j}}{\partial x^{l} \partial t}+\frac{\partial g_{m l}}{\partial x^{j} \partial t}-\frac{\partial g_{j l}}{\partial x^{m} \partial t}\right)-g_{h k} \frac{\partial^{2} g^{h m}}{\partial x^{j} \partial t}\left(\frac{\partial g_{m i}}{\partial x^{l} \partial t}+\frac{\partial g_{m l}}{\partial x^{i} \partial t}-\frac{\partial g_{i l}}{\partial x^{m} \partial t}\right) \\
& +g_{h k} \frac{\partial g^{h m}}{\partial t}\left[\frac{\partial}{\partial x^{i}}\left(\frac{\partial g_{m j}}{\partial x^{l} \partial t}+\frac{\partial g_{m l}}{\partial x^{j} \partial t}-\frac{\partial g_{j l}}{\partial x^{m} \partial t}\right)-\frac{\partial}{\partial x^{j}}\left(\frac{\partial^{2} g_{m i}}{\partial x^{l} \partial t}+\frac{\partial^{2} g_{m l}}{\partial x^{i} \partial t}-\frac{\partial^{2} g_{i l}}{\partial x^{m} \partial t}\right)\right] \\
& +\frac{1}{2}\left[\frac{\partial^{2}}{\partial x^{i} \partial x^{l}}\left(\frac{\partial^{2} g_{k j}}{\partial t^{2}}\right)+\frac{\partial^{2}}{\partial x^{i} \partial x^{j}}\left(\frac{\partial^{2} g_{k l}}{\partial t^{2}}\right)-\frac{\partial^{2}}{\partial x^{i} \partial x^{k}}\left(\frac{\partial^{2} g_{j l}}{\partial t^{2}}\right)\right] \\
& -\frac{1}{2}\left[\frac{\partial^{2}}{\partial x^{j} \partial x^{l}}\left(\frac{\partial^{2} g_{k i}}{\partial t^{2}}\right)+\frac{\partial^{2}}{\partial x^{j} \partial x^{i}}\left(\frac{\partial^{2} g_{k l}}{\partial t^{2}}\right)-\frac{\partial^{2}}{\partial x^{j} \partial x^{k}}\left(\frac{\partial^{2} g_{i l}}{\partial t^{2}}\right)\right] \\
& +2 g_{h k}\left(\frac{\partial}{\partial t} \Gamma_{i p}^{h} \frac{\partial}{\partial t} \Gamma_{j l}^{p}-\frac{\partial}{\partial t} \Gamma_{j p}^{h} \frac{\partial}{\partial t} \Gamma_{i l}^{p}\right)
\end{aligned}
$$




$$
\begin{aligned}
& +\frac{\partial g_{h k}}{\partial t} \frac{\partial g^{h m}}{\partial t}\left[\frac{\partial}{\partial x^{i}}\left(\frac{\partial g_{m j}}{\partial x^{l}}+\frac{\partial g_{m l}}{\partial x^{j}}-\frac{\partial g_{j l}}{\partial x^{m}}\right)-\frac{\partial}{\partial x^{j}}\left(\frac{\partial g_{m i}}{\partial x^{l}}+\frac{\partial g_{m l}}{\partial x^{i}}-\frac{\partial g_{i l}}{\partial x^{m}}\right)\right] \\
& +\frac{\partial g_{h k}}{\partial t} g^{h m}\left[\frac{\partial}{\partial x^{i}}\left(\frac{\partial g_{m j}}{\partial x^{l} \partial t}+\frac{\partial g_{m l}}{\partial x^{j} \partial t}-\frac{\partial g_{j l}}{\partial x^{m} \partial t}\right)-\frac{\partial}{\partial x^{j}}\left(\frac{\partial g_{m i}}{\partial x^{l} \partial t}+\frac{\partial g_{m l}}{\partial x^{i} \partial t}-\frac{\partial g_{i l}}{\partial x^{m} \partial t}\right)\right] \\
& +R_{i j l}^{h} \frac{\partial^{2} g_{h k}}{\partial t^{2}} .
\end{aligned}
$$

Noting $g^{h m} g_{m l}=\delta_{l}^{h}$, we get

$$
\begin{aligned}
& \frac{\partial g^{h m}}{\partial t}=-g^{h p} g^{m q} \frac{\partial g_{p q}}{\partial t}, \quad \frac{\partial^{2} g^{h m}}{\partial x^{k} \partial t}=-g^{h p} g^{m q} \frac{\partial g_{p q}}{\partial x^{k} \partial t}, \\
& \frac{\partial^{2} g^{h m}}{\partial t^{2}}=-g^{h p} g^{m q} \frac{\partial^{2} g_{p q}}{\partial t^{2}}+2 g^{h p} g^{r q} g^{s m} \frac{\partial g_{p q}}{\partial t} \frac{\partial g_{r s}}{\partial t} .
\end{aligned}
$$

Thus, it follows from (5.2) that

$$
\begin{aligned}
& \frac{\partial^{2}}{\partial t^{2}} R_{i j k l}=\left(-\frac{1}{2} g^{p m} \frac{\partial^{2} g_{k p}}{\partial t^{2}}+g^{r q} g^{p m} \frac{\partial g_{k q}}{\partial t} \frac{\partial g_{r p}}{\partial t}\right) \\
\times & {\left[\frac{\partial}{\partial x^{i}}\left(\frac{\partial g_{m j}}{\partial x^{l}}+\frac{\partial g_{m l}}{\partial x^{j}}-\frac{\partial g_{j l}}{\partial x^{m}}\right)-\frac{\partial}{\partial x^{j}}\left(\frac{\partial g_{m i}}{\partial x^{l}}+\frac{\partial g_{m l}}{\partial x^{i}}-\frac{\partial g_{i l}}{\partial x^{m}}\right)\right]-g^{p m} \frac{\partial^{2} g_{k p}}{\partial x^{i} \partial t} } \\
& \cdot\left(\frac{\partial^{2} g_{m j}}{\partial x^{l} \partial t}+\frac{\partial^{2} g_{m l}}{\partial x^{j} \partial t}-\frac{\partial^{2} g_{j l}}{\partial x^{m} \partial t}\right)+g^{p m} \frac{\partial^{2} g_{k p}}{\partial x^{j} \partial t}\left(\frac{\partial^{2} g_{m i}}{\partial x^{l} \partial t}+\frac{\partial^{2} g_{m l}}{\partial x^{i} \partial t}-\frac{\partial^{2} g_{i l}}{\partial x^{m} \partial t}\right) \\
+ & \frac{1}{2}\left[\frac{\partial^{2}}{\partial x^{i} \partial x^{l}}\left(\frac{\partial^{2} g_{k j}}{\partial t^{2}}\right)+\frac{\partial^{2}}{\partial x^{i} \partial x^{j}}\left(\frac{\partial^{2} g_{k l}}{\partial t^{2}}\right)-\frac{\partial^{2}}{\partial x^{i} \partial x^{k}}\left(\frac{\partial^{2} g_{j l}}{\partial t^{2}}\right)\right] \\
- & \frac{1}{2}\left[\frac{\partial^{2}}{\partial x^{j} \partial x^{l}}\left(\frac{\partial^{2} g_{k i}}{\partial t^{2}}\right)+\frac{\partial^{2}}{\partial x^{j} \partial x^{i}}\left(\frac{\partial^{2} g_{k l}}{\partial t^{2}}\right)-\frac{\partial^{2}}{\partial x^{j} \partial x^{k}}\left(\frac{\partial^{2} g_{i l}}{\partial t^{2}}\right)\right] \\
+ & 2 g_{h k}\left(\frac{\partial}{\partial t} \Gamma_{i p}^{h} \cdot \frac{\partial}{\partial t} \Gamma_{j l}^{p}-\frac{\partial}{\partial t} \Gamma_{j p}^{h} \cdot \frac{\partial}{\partial t} \Gamma_{i l}^{p}\right)+R_{i j l}^{h} \frac{\partial^{2} g_{h k}}{\partial t^{2}}-g^{h p} g^{m q} \frac{\partial g_{n k}}{\partial t} \frac{\partial g_{p q}}{\partial t} \\
& \cdot\left[\frac{\partial}{\partial x^{i}}\left(\frac{\partial g_{m j}}{\partial x^{l}}+\frac{\partial g_{m l}}{\partial x^{j}}-\frac{\partial g_{j l}}{\partial x^{m}}\right)-\frac{\partial}{\partial x^{j}}\left(\frac{\partial g_{m i}}{\partial x^{l}}+\frac{\partial g_{m l}}{\partial x^{i}}-\frac{\partial g_{i l}}{\partial x^{m}}\right)\right] \\
= & -\frac{\partial^{2} g_{k p}}{\partial t^{2}}\left(\frac{\partial}{\partial x^{i}} \Gamma_{j l}^{p}-\frac{\partial}{\partial x^{j}} \Gamma_{i l}^{p}\right)+2 g^{r q} \frac{\partial g_{k q}}{\partial t} \frac{\partial g_{r p}}{\partial t}\left(\frac{\partial}{\partial x^{i}} \Gamma_{j l}^{p}-\frac{\partial}{\partial x^{j}} \Gamma_{i l}^{p}\right) \\
& -\frac{\partial^{2} g_{k p}}{\partial x^{i} \partial t} \cdot g^{p m}\left(\frac{\partial^{2} g_{m j}}{\partial x^{l} \partial t}+\frac{\partial^{2} g_{m l}}{\partial x^{j} \partial t}-\frac{\partial^{2} g_{j l}}{\partial x^{m} \partial t}\right) \\
& +\frac{\partial^{2} g_{k p}}{\partial x^{j} \partial t} \cdot g^{p m}\left(\frac{\partial^{2} g_{m i}}{\partial x^{l} \partial t}+\frac{\partial^{2} g_{m l}}{\partial x^{i} \partial t}-\frac{\partial^{2} g_{i l}}{\partial x^{m} \partial t}\right)
\end{aligned}
$$




$$
\begin{aligned}
+ & \frac{1}{2}\left[\frac{\partial^{2}}{\partial x^{i} \partial x^{l}}\left(-2 R_{k j}\right)+\frac{\partial^{2}}{\partial x^{i} \partial x^{j}}\left(-2 R_{k l}\right)-\frac{\partial^{2}}{\partial x^{i} \partial x^{k}}\left(-2 R_{j l}\right)\right] \\
& -\frac{1}{2}\left[\frac{\partial^{2}}{\partial x^{j} \partial x^{l}}\left(-2 R_{i k}\right)+\frac{\partial^{2}}{\partial x^{i} \partial x^{j}}\left(-2 R_{k l}\right)-\frac{\partial^{2}}{\partial x^{j} \partial x^{k}}\left(-2 R_{i l}\right)\right] \\
& +2 g_{h k}\left[\frac{\partial}{\partial t} \Gamma_{i p}^{h} \cdot \frac{\partial}{\partial t} \Gamma_{j l}^{p}-\frac{\partial}{\partial t} \Gamma_{j p}^{h} \cdot \frac{\partial}{\partial t} \Gamma_{i l}^{p}\right]-\frac{\partial g_{h k}}{\partial t} \frac{\partial g_{p q}}{\partial t} g^{h p} \\
& \cdot 2\left(\frac{\partial}{\partial x_{i}} \Gamma_{j l}^{q}-\frac{\partial}{\partial x_{j}} \Gamma_{i l}^{q}\right)+R_{i j l}^{h} \frac{\partial^{2} g_{h k}}{\partial t^{2}} \\
= & \frac{1}{2}\left[\frac{\partial^{2}}{\partial x^{i} \partial x^{l}}\left(-2 R_{k j}\right)+\frac{\partial^{2}}{\partial x^{i} \partial x^{j}}\left(-2 R_{k l}\right)-\frac{\partial^{2}}{\partial x^{i} \partial x^{k}}\left(-2 R_{j l}\right)\right] \\
& -\frac{1}{2}\left[\frac{\partial^{2}}{\partial x^{j} \partial x^{l}}\left(-2 R_{k i}\right)+\frac{\partial^{2}}{\partial x^{i} \partial x^{j}}\left(-2 R_{k l}\right)-\frac{\partial^{2}}{\partial x^{j} \partial x^{k}}\left(-2 R_{i l}\right)\right]-g^{p m} \frac{\partial^{2} g_{k p}}{\partial x^{i} \partial t} \\
& \cdot\left(\frac{\partial^{2} g_{m j}}{\partial x^{l} \partial t}+\frac{\partial^{2} g_{m l}}{\partial x^{j} \partial t}-\frac{\partial^{2} g_{j l}}{\partial x^{m} \partial t}\right)+g^{p m} \frac{\partial^{2} g_{k p}}{\partial x^{j} \partial t}\left(\frac{\partial^{2} g_{m i}}{\partial x^{l} \partial t}+\frac{\partial^{2} g_{m l}}{\partial x^{i} \partial t}-\frac{\partial^{2} g_{i l}}{\partial x^{m} \partial t}\right) \\
& +2 g_{h k}\left(\frac{\partial}{\partial t} \Gamma_{i p}^{h} \cdot \frac{\partial}{\partial t} \Gamma_{j l}^{p}-\frac{\partial}{\partial t} \Gamma_{j p}^{h} \cdot \frac{\partial}{\partial t} \Gamma_{i l}^{p}\right) \cdot
\end{aligned}
$$

On the one hand, we have

$$
\frac{\partial^{2}}{\partial x^{i} \partial x^{l}} R_{j k}=\nabla_{i} \nabla_{l} R_{j k}+\nabla_{i} \Gamma_{l k}^{p} \cdot R_{j p}+\nabla_{i} \Gamma_{l j}^{p} \cdot R_{k p} .
$$

Then

$$
\begin{aligned}
& \frac{1}{2}\left[\frac{\partial^{2}}{\partial x^{i} \partial x^{l}}\left(-2 R_{k j}\right)+\frac{\partial^{2}}{\partial x^{i} \partial x^{j}}\left(-2 R_{k l}\right)-\frac{\partial^{2}}{\partial x^{i} \partial x^{k}}\left(-2 R_{j l}\right)\right] \\
& -\frac{1}{2}\left[\frac{\partial^{2}}{\partial x^{j} \partial x^{l}}\left(-2 R_{k i}\right)+\frac{\partial^{2}}{\partial x^{i} \partial x^{j}}\left(-2 R_{k l}\right)-\frac{\partial^{2}}{\partial x^{j} \partial x^{k}}\left(-2 R_{i l}\right)\right] \\
= & -\nabla_{i} \nabla_{l} R_{k j}-\nabla_{i} \Gamma_{l k}^{p} R_{j p}-\nabla_{i} \Gamma_{l j}^{p} R_{k p}-\nabla_{i} \nabla_{j} R_{k l}-\nabla_{i} \Gamma_{j k}^{p} R_{l p}-\nabla_{i} \Gamma_{j l}^{p} R_{k p} \\
& +\nabla_{i} \nabla_{k} R_{j l}+\nabla_{i} \Gamma_{k j}^{p} R_{l p}+\nabla_{i} \Gamma_{k l}^{p} R_{j p}+\nabla_{i} \nabla_{l} R_{k i}+\nabla_{j} \Gamma_{k l}^{p} R_{i p}+\nabla_{j} \Gamma_{l i}^{p} R_{k p} \\
& +\nabla_{j} \nabla_{i} R_{k l}+\nabla_{j} \Gamma_{i k}^{p} R_{l p}+\nabla_{j} \Gamma_{i l}^{p} R_{k p}-\nabla_{j} \nabla_{k} R_{i l}-\nabla_{j} \Gamma_{k i}^{p} R_{p l}-\nabla_{j} \Gamma_{k l}^{p} R_{p i} \\
= & -\nabla_{i} \nabla_{l} R_{k j}-\nabla_{i} \nabla_{j} R_{k l}+\nabla_{i} \nabla_{k} R_{j l}+\nabla_{j} \nabla_{l} R_{k i}+\nabla_{j} \nabla_{i} R_{k i}-\nabla_{j} \nabla_{k} R_{i l} \\
& +R_{i p}\left(\nabla_{j} \Gamma_{l k}^{p}-\nabla_{j} \Gamma_{k l}^{p}\right)+R_{j p}\left(-\nabla_{i} \Gamma_{l k}^{p}+\nabla_{i} \Gamma_{k l}^{p}\right) \\
& +R_{k p}\left(-\nabla_{i} \Gamma_{l j}^{p}-\nabla_{i} \Gamma_{j l}^{p}+\nabla_{j} \Gamma_{l i}^{p}+\nabla_{j} \Gamma_{i l}^{p}\right)
\end{aligned}
$$




$$
\begin{aligned}
& +R_{l p}\left(-\nabla_{i} \Gamma_{j k}^{p}+\nabla_{i} \Gamma_{k j}^{p}+\nabla_{j} \Gamma_{i k}^{p}-\nabla_{j} \Gamma_{k i}^{p}\right) \\
= & -\nabla_{i} \nabla_{l} R_{k j}+\nabla_{i} \nabla_{k} R_{j l}+\nabla_{j} \nabla_{l} R_{k i}-\nabla_{j} \nabla_{k} R_{i l} \\
& -R_{i j l p} g^{p q} R_{q k}-R_{i j k p} g^{p q} R_{q l}+R_{k p}\left(-2 R_{i j l}^{p}\right) \\
= & -\nabla_{i} \nabla_{l} R_{k j}+\nabla_{i} \nabla_{k} R_{j l}+\nabla_{j} \nabla_{l} R_{k i}-\nabla_{j} \nabla_{k} R_{i l}-g^{p q}\left(R_{i j l p} R_{k q}+R_{i j k p} R_{l q}\right) .
\end{aligned}
$$

On the other hand, we have

$$
\begin{aligned}
& -g^{p m} \frac{\partial^{2} g_{k p}}{\partial x^{i} \partial t}\left(\frac{\partial^{2} g_{m j}}{\partial x^{l} \partial t}+\frac{\partial^{2} g_{m l}}{\partial x^{j} \partial t}-\frac{\partial^{2} g_{j l}}{\partial x^{m} \partial t}\right)+g^{p m} \frac{\partial^{2} g_{k p}}{\partial x^{j} \partial t} \\
& \cdot\left(\frac{\partial^{2} g_{m i}}{\partial x^{l} \partial t}+\frac{\partial^{2} g_{m l}}{\partial x^{i} \partial t}-\frac{\partial^{2} g_{i l}}{\partial x^{m} \partial t}\right)+2 g_{h k}\left(\frac{\partial}{\partial t} \Gamma_{i p}^{h} \cdot \frac{\partial}{\partial t} \Gamma_{j l}^{p}-\frac{\partial}{\partial t} \Gamma_{j p}^{h} \cdot \frac{\partial}{\partial t} \Gamma_{i l}^{p}\right) \\
= & -g^{p m} \frac{\partial^{2} g_{k p}}{\partial x^{i} \partial t}\left(\frac{\partial^{2} g_{m j}}{\partial x^{l} \partial t}+\frac{\partial^{2} g_{m l}}{\partial x^{j} \partial t}-\frac{\partial^{2} g_{j l}}{\partial x^{m} \partial t}\right) \\
& +g^{p m} \frac{\partial^{2} g_{k p}}{\partial x^{j} \partial t}\left(\frac{\partial^{2} g_{m i}}{\partial x^{l} \partial t}+\frac{\partial^{2} g_{m l}}{\partial x^{i} \partial t}-\frac{\partial^{2} g_{i l}}{\partial x^{m} \partial t}\right) \\
& +\frac{1}{2} g^{p m}\left(\frac{\partial^{2} g_{k i}}{\partial x^{p} \partial t}+\frac{\partial^{2} g_{k p}}{\partial x^{i} \partial t}-\frac{\partial^{2} g_{i p}}{\partial x^{k} \partial t}\right)\left(\frac{\partial^{2} g_{m j}}{\partial x^{l} \partial t}+\frac{\partial^{2} g_{m l}}{\partial x^{j} \partial t}-\frac{\partial^{2} g_{j l}}{\partial x^{m} \partial t}\right) \\
& -\frac{1}{2} g^{p m}\left(\frac{\partial^{2} g_{k j}}{\partial x^{p} \partial t}+\frac{\partial^{2} g_{k p}}{\partial x^{j} \partial t}-\frac{\partial^{2} g_{j p}}{\partial x^{k} \partial t}\right)\left(\frac{\partial^{2} g_{m i}}{\partial x^{l} \partial t}+\frac{\partial^{2} g_{m l}}{\partial x^{i} \partial t}-\frac{\partial^{2} g_{i l}}{\partial x^{m} \partial t}\right) \\
= & g^{p m}\left\{\frac{1}{2}\left(\frac{\partial^{2} g_{m j}}{\partial x^{l} \partial t}+\frac{\partial^{2} g_{m l}}{\partial x^{j} \partial t}-\frac{\partial^{2} g_{j l}}{\partial x^{m} \partial t}\right)\left(\frac{\partial^{2} g_{k i}}{\partial x^{p} \partial t}-\frac{\partial^{2} g_{i p}}{\partial x^{k} \partial t}-\frac{\partial^{2} g_{k p}}{\partial x^{i} \partial t}\right)\right. \\
& \left.-\frac{1}{2}\left(\frac{\partial^{2} g_{m i}}{\partial x^{l} \partial t}+\frac{\partial^{2} g_{m l}}{\partial x^{i} \partial t}-\frac{\partial^{2} g_{i l}}{\partial x^{m} \partial t}\right)\left(\frac{\partial^{2} g_{k j}}{\partial x^{p} \partial t}-\frac{\partial^{2} g_{j p}}{\partial x^{k} \partial t}-\frac{\partial^{2} g_{k p}}{\partial x^{j} \partial t}\right)\right\} \\
= & 2 g_{p q}\left\{\frac{1}{2} g^{p r} \frac{\partial}{\partial t}\left(\frac{\partial g_{r j}}{\partial x^{l}}+\frac{\partial g_{r l}}{\partial x^{j}}-\frac{\partial g_{j l}}{\partial x^{r}}\right) \cdot \frac{1}{2} g^{q s} \frac{\partial}{\partial t}\left(\frac{\partial g_{k i}}{\partial x^{s}}-\frac{\partial g_{i s}}{\partial x^{k}}-\frac{\partial g_{k s}}{\partial x^{i}}\right)\right. \\
& \left.-\frac{1}{2} g^{p r} \frac{\partial}{\partial t}\left(\frac{\partial g_{r i}}{\partial x^{l}}+\frac{\partial g_{r l}}{\partial x^{i}}-\frac{\partial g_{i l}}{\partial x^{r}}\right) \cdot \frac{1}{2} g^{q s} \frac{\partial}{\partial t}\left(\frac{\partial g_{k j}}{\partial x^{s}}-\frac{\partial g_{j s}}{\partial x^{k}}-\frac{\partial g_{k s}}{\partial x^{j}}\right)\right\} \\
= & 2 g_{p q}\left(\frac{\partial}{\partial t} \Gamma_{i l}^{p} \cdot \frac{\partial}{\partial t} \Gamma_{j k}^{q}-\frac{\partial}{\partial t} \Gamma_{j l}^{p} \cdot \frac{\partial}{\partial t} \Gamma_{i k}^{q}\right) \cdot \\
& \\
&
\end{aligned}
$$

Therefore, it follows from (5.3), (5.4) and (5.5) that

$$
\begin{aligned}
\frac{\partial^{2}}{\partial t^{2}} R_{i j k l}= & -\nabla_{i} \nabla_{l} R_{k j}+\nabla_{i} \nabla_{k} R_{j l}+\nabla_{j} \nabla_{l} R_{k i}-\nabla_{j} \nabla_{k} R_{i l} \\
& -g^{p q}\left(R_{i j q l} R_{k p}+R_{i j k q} R_{k p}\right)+2 g_{p q}\left(\frac{\partial}{\partial t} \Gamma_{i l}^{p} \cdot \frac{\partial}{\partial t} \Gamma_{j k}^{q}-\frac{\partial}{\partial t} \Gamma_{j l}^{p} \cdot \frac{\partial}{\partial t} \Gamma_{i k}^{q}\right) .
\end{aligned}
$$


Similar to Hamilton [6], we have

Theorem 5.1. Under the hyperbolic geometric flow (5.1), the Riemannian curvature tensor $R_{i j k l}$ satisfies the evolution equation

$$
\begin{aligned}
\frac{\partial^{2}}{\partial t^{2}} R_{i j k l}= & \triangle R_{i j k l}+2\left(B_{i j k l}-B_{i j l k}-B_{i l j k}+B_{i k j l}\right) \\
& -g^{p q}\left(R_{p j k l} R_{q i}+R_{i p k l} R_{q j}+R_{i j p l} R_{q k}+R_{i j k p} R_{q l}\right) \\
& +2 g_{p q}\left(\frac{\partial}{\partial t} \Gamma_{i l}^{p} \cdot \frac{\partial}{\partial t} \Gamma_{j k}^{q}-\frac{\partial}{\partial t} \Gamma_{j l}^{p} \cdot \frac{\partial}{\partial t} \Gamma_{i k}^{q}\right)
\end{aligned}
$$

where $B_{i j k l}=g^{p r} g^{q s} R_{p i q j} R_{r k s l}$ and $\triangle$ is the Laplacian with respect to the evolving metric.

Remark 5.1. In Theorem 5.1 and Theorem 5.2 below, the term

$$
2 g_{p q}\left(\frac{\partial}{\partial t} \Gamma_{i l}^{p} \cdot \frac{\partial}{\partial t} \Gamma_{j k}^{q}-\frac{\partial}{\partial t} \Gamma_{j l}^{p} \cdot \frac{\partial}{\partial t} \Gamma_{i k}^{q}\right)
$$

can be written in the covariant form. For the sake of simplicity, we omit it.

For the Ricci curvature tensor, we have

$$
\begin{aligned}
\frac{\partial^{2}}{\partial t^{2}} R_{i k} & =\frac{\partial^{2}}{\partial t^{2}}\left(R_{i j k l} g^{j l}\right) \\
& =g^{j l} \frac{\partial^{2}}{\partial t^{2}} R_{i j k l}+2 \frac{\partial}{\partial t} g^{j l} \cdot \frac{\partial}{\partial t} R_{i j k l}+R_{i j k l} \frac{\partial^{2} g^{j l}}{\partial t^{2}} \\
& =g^{j l} \frac{\partial^{2}}{\partial t^{2}} R_{i j k l}-2 g^{j p} g^{l q} \frac{\partial g_{p q}}{\partial t} \frac{\partial}{\partial t} R_{i j k l}-g^{j p} g^{l q} \frac{\partial^{2} g_{p q}}{\partial t^{2}} R_{i j k l} \\
& +2 g^{j p} g^{r q} g^{s l} \frac{\partial g_{p q}}{\partial t} \frac{\partial g_{r s}}{\partial t} R_{i j k l} .
\end{aligned}
$$

Thus, we obtain

Theorem 5.2. Under the hyperbolic geometric flow (5.1), the Ricci curvature tensor satisfies

$$
\begin{aligned}
\frac{\partial^{2}}{\partial t^{2}} R_{i k}= & \triangle R_{i k}+2 g^{p r} g^{q s} R_{p i q k} R_{r s}-2 g^{p q} R_{p i} R_{q k} \\
& +2 g^{j l} g_{p q}\left(\frac{\partial}{\partial t} \Gamma_{i l}^{p} \frac{\partial}{\partial t} \Gamma_{j k}^{q}-\frac{\partial}{\partial t} \Gamma_{j l}^{p} \frac{\partial}{\partial t} \Gamma_{i k}^{q}\right) \\
& -2 g^{j p} g^{l q} \frac{\partial g_{p q}}{\partial t} \frac{\partial}{\partial t} R_{i j k l}+2 g^{j p} g^{r q} g^{s l} \frac{\partial g_{p q}}{\partial t} \frac{\partial g_{r s}}{\partial t} R_{i j k l}
\end{aligned}
$$


For the scalar curvature, we have

$$
\begin{aligned}
\frac{\partial^{2}}{\partial t^{2}} R & =\frac{\partial^{2}}{\partial t^{2}}\left(g^{i k} R_{i k}\right) \\
& =g^{i k} \frac{\partial^{2}}{\partial t^{2}} R_{i k}+2 \frac{\partial}{\partial t} R_{i k} \cdot \frac{\partial}{\partial t} g^{i k}+R_{i k} \frac{\partial^{2} g^{i k}}{\partial t^{2}} \\
& =g^{i k} \frac{\partial^{2}}{\partial t^{2}} R_{i k}-2 g^{i p} g^{k q} \frac{\partial g_{p q}}{\partial t} \frac{\partial R_{i k}}{\partial t}+R_{i k}\left(-g^{i p} g^{k q} \frac{\partial^{2} g_{p q}}{\partial t^{2}}+2 g^{i p} g^{r q} g^{s k} \frac{\partial g_{p q}}{\partial t} \frac{\partial g_{r s}}{\partial t}\right) .
\end{aligned}
$$

On the other hand,

$$
\begin{aligned}
& 2 g^{i k} g^{j l} g_{p q}\left(\frac{\partial}{\partial t} \Gamma_{i l}^{p} \frac{\partial}{\partial t} \Gamma_{j k}^{q}-\frac{\partial}{\partial t} \Gamma_{j l}^{p} \frac{\partial}{\partial t} \Gamma_{i k}^{q}\right) \\
= & \frac{3}{2} g^{i k} g^{j l} g^{r s} \nabla_{r}\left(\frac{\partial g_{i j}}{\partial t}\right) \nabla_{s}\left(\frac{\partial g_{k l}}{\partial t}\right)-\frac{1}{2} g^{r s} \nabla_{r}\left(g^{i k} \frac{\partial g_{i k}}{\partial t}\right) \nabla_{s}\left(g^{j l} \frac{\partial g_{j l}}{\partial t}\right) \\
& +2 g^{r s} g^{j l} \nabla_{r}\left(g^{i k} \frac{\partial g_{i k}}{\partial t}\right) \nabla_{l}\left(\frac{\partial g_{j s}}{\partial t}\right)-g^{i k} g^{j l} g^{r s} \nabla_{r}\left(\frac{\partial g_{i j}}{\partial t}\right) \nabla_{l}\left(\frac{\partial g_{k s}}{\partial t}\right) \\
- & 2 g^{i k} g^{j l} g^{r s} \nabla_{i}\left(\frac{\partial g_{k r}}{\partial t}\right) \nabla_{j}\left(\frac{\partial g_{l s}}{\partial t}\right) .
\end{aligned}
$$

Then, we get

Theorem 5.3. Under the hyperbolic geometric flow (5.1), the scalar curvature satisfies

$$
\begin{aligned}
\frac{\partial^{2}}{\partial t^{2}} R= & \triangle R+2|\operatorname{Ric}|^{2} \\
& +\frac{3}{2} g^{i k} g^{j l} g^{r s} \nabla_{r}\left(\frac{\partial g_{i j}}{\partial t}\right) \nabla_{s}\left(\frac{\partial g_{k l}}{\partial t}\right)-\frac{1}{2} g^{r s} \nabla_{r}\left(g^{i k} \frac{\partial g_{i k}}{\partial t}\right) \nabla_{s}\left(g^{j l} \frac{\partial g_{j l}}{\partial t}\right) \\
& +2 g^{r s} g^{j l} \nabla_{r}\left(g^{i k} \frac{\partial g_{i k}}{\partial t}\right) \nabla_{l}\left(\frac{\partial g_{j s}}{\partial t}\right)-g^{i k} g^{j l} g^{r s} \nabla_{r}\left(\frac{\partial g_{i j}}{\partial t}\right) \nabla_{l}\left(\frac{\partial g_{k s}}{\partial t}\right) \\
& -2 g^{i k} g^{j l} g^{r s} \nabla_{i}\left(\frac{\partial g_{k r}}{\partial t}\right) \nabla_{j}\left(\frac{\partial g_{l s}}{\partial t}\right)-2 g^{i k} g^{j p} g^{l q} \frac{\partial g_{p q}}{\partial t} \frac{\partial}{\partial t} R_{i j k l} \\
& -2 g^{i p} g^{k q} \frac{\partial g_{p q}}{\partial t} \frac{\partial R_{i k}}{\partial t}+4 R_{i k} g^{i p} g^{r q} g^{s k} \frac{\partial g_{p q}}{\partial t} \frac{\partial g_{r s}}{\partial t} .
\end{aligned}
$$

Theorems 5.1-5.3 show that the curvatures of the hyperbolic geometric flow possess the wave character. We will apply techniques from hyperbolic equations to the above wave equations of curvatures to derive various geometric results. 


\section{Discussions}

The hyperbolic geometric flow describes the wave character of the metrics and curvatures of manifolds. Many hyperbolic systems in nature provide natural singular sets, the typical example is the Einstein equations in general relativity which form a hyperbolic system with a well-posed Cauchy problem. If one starts with smooth initial data, one may end up with a singular space-time. One of the most challenging problems is to describe the kind of natural singularity. The famous cosmic censorship conjecture due to Penrose is an attempt to describe such singularities (see [12]). In Kong and Liu [11], we construct some exact solutions of the hyperbolic geometric flow, these solutions possess the singularities which are nothing but those described by Penrose's conjecture. By these examples, we believe that the hyperbolic geometric flow is a very natural and powerful tool to understand the singularities in the nature, in particular, the singularity described by Penrose cosmic censorship conjecture.

The Einstein equations play an essential role in general relativity. Consider a space-time with Lorentzian metric

$$
d s^{2}=g_{\mu \nu} d x^{\mu} d x^{\nu} \quad(\mu, \nu=0,1,2 \cdots, n) .
$$

The vacuum Einstein equations read

$$
G_{\mu \nu}=0
$$

where $G_{\mu \nu}$ is the Einstein tensor. We now consider the following metric with orthogonal time-axis

$$
d s^{2}=-d t^{2}+g_{i j}(x, t) d x^{i} d x^{j} .
$$

Substituting (6.3) into (6.2), we can obtain the equations satisfied by the metric $g_{i j}$

$$
\frac{\partial^{2} g_{i j}}{\partial t^{2}}=-2 R_{i j}-\frac{1}{2} g^{p q} \frac{\partial g_{i j}}{\partial t} \frac{\partial g_{p q}}{\partial t}+g^{p q} \frac{\partial g_{i p}}{\partial t} \frac{\partial g_{j q}}{\partial t} .
$$

Neglecting the lower order terms gives the hyperbolic geometric flow (1.1). Therefore, in this sense, the hyperbolic geometric flow can be viewed as the leading terms in the vacuum Einstein equations with respect to the metric (6.3). Since the hyperbolic geometric flow only contains the main terms in the Einstein equations, it not only becomes simpler and more symmetric, but also possesses rich and beautiful geometric properties. In particular, in mathematics, its Cauchy problem is well-posed and easier to handle some fundamental problems such as 
the global existence and formation of singularities; on the other hand, it can be applied to re-understand the singularity of the universe and other important problems in physics and cosmology (see [16]). We also believe that there should be some relations between the solutions of the Einstein equations and the corresponding hyperbolic geometric flows. On the other hand, from the above discussions we have seen that the hyperbolic geometric flow also possesses many beautiful features similar to those of the Ricci flow, and some of the techniques in the study of the Ricci flow can be directly used to understand the hyperbolic geometric flow. The deep study on the hyperbolic geometric flow may open a new way to understand the complicated Einstein equations.

It is well known, in general relativity there is a constraint system of equations involving an asymptotically flat metric tensor and another symmetric tensor. There are four constraint equations and it is therefore over-determined. Unlike this, since the time axis is orthogonal to other space axes, the hyperbolic geometric flow does not need to satisfy any additional constraint. More precisely, for the Cauchy problem of the hyperbolic geometric flow, in order to determine the solution we need two initial conditions: one is the metric flow itself $g_{i j}(x, 0)$, another is its derivative $\frac{\partial g_{i j}}{\partial t}(x, 0)$, since the time axis is orthogonal to other axes, these initial data do not need to satisfy any additional constraint, and therefore it is a determined system. This is another main new feature of the hyperbolic geometric flow.

Many mathematicians, for example Shatah et al [13]-[15], have investigated the Cauchy problem for some geometric wave equations. The model at hand is the harmonic map problem, which is the study of maps from the Minkowski space-time into complete Riemannian manifolds. This kind of geometric wave equations is a system of partial differential equations of second order, which is the Euler-Lagrange equations of the action integral of the harmonic map. It satisfies certain linear matching condition, and then under suitable assumptions, has a unique small smooth solution for all time, and possesses some interesting (decay, energy and regularity) estimates. On the other hand, the hyperbolic geometric flow is determined by the Ricci curvatures of a family of Riemannian metrics on the manifold under consideration. That is to say, the hyperbolic geometric flow possesses itself intrinsic geometric structure and can be used to describe the wave character of metrics and curvatures. This is essentially different from the above harmonic map problem. 
As well-known, one can understand the heat kernel from the kernel of wave equation. This indicates that we should be able to derive various information of the Ricci flow from that of the hyperbolic geometric flow. Therefore it is also interesting to understand the relations between the hyperbolic geometric flow and the Ricci flow, the singularities of its solutions and its relation with the geometrization theorem. This will be another interesting topic in the sequel.

Acknowledgements. The authors thank the referee for valuable suggestions. The work of Dai was supported by the fund of post-doctor of China (Grant No. 20060401063); the work of Kong was supported in part by the NNSF of China (Grant No. 10671124) and the Qiu-Shi Professor Fellowship from Zhejiang University; the work of Liu was supported in part by the NSF and NSF of China.

\section{REFERENCES}

[1] Huaidong Cao and Xiping Zhu, A complete proof of the Poincaré and geometrization conjectures - application of the Hamilton-Perelman theory of the Ricci flow, Asian J. Math. 10 (2006), 165-492.

[2] D. Christodoulou, Global solutions of nonlinear hyperbolic equations for small initial data, Comm. Pure Appl. Math. 39 (1986), 367-282.

[3] A.E. Fischer and J.E. Marsden, The Einstein evolution equations as a first-order quasilinear symmetric system hyperbolic system I, Commun. Math. Phys. 28 (1972), 1-38.

[4] V. Fock, The theory of space, time and gravitation, second revised edition. Translated from the Russian by N. Kemmer. A Pergamon Press Book The Macmillan Co., New York, 1964.

[5] K.O. Friedrich, Symmetric hyperbolic linear differential equations, Comm. Pure Appl. Math. 7 (1954), 345-392.

[6] R. Hamilton, Three-manifolds with positive Ricci curvature, J. Differential Geom. 17 (1982), 255-306.

[7] L. Hörmander, Lectures on nonlinear hyperbolic differential equations, Mathématiques \& Applications 26, Springer-Verlag, Berlin, 1997.

[8] F. John, Delayed singularity formation in solutions of nonlinear wave equations in higher dimensions, Comm. Pure Appl. Math. 29 (1976), 649-682.

[9] T. Kato, Quasilinear equations of evolution with applications to partial differential equations, Springer Lecture Notes 448, 1975, 25-70.

[10] S. Klainerman, Global existence for nonlinear wave equations, Comm. Pure Appl. Math. 33 (1980), 43-101.

[11] De-Xing Kong and Kefeng Liu, Wave character of metrics and hyperbolic geometric flow, J. Math. Phys. 48 (2007), 103508-1-103508-14. 
[12] R. Penrose, Gravitational collapse and space-time singularities, Phys. Rev. Lett. 14 (1965), $57-59$.

[13] J. Shatah and M. Struwe, Geometric Wave Equations, Courant Lecture Notes in Mathematics 2, New York University, Courant Institute of Mathematical Sciences, New York; American Mathematical Society, Providence, RI, 1998.

[14] J. Shatah and M. Struwe, Regularity results for nonlinear wave equations, Ann. of Math. 138 (1993), 503-518.

[15] J. Shatah and A. Tahvildar-Zadeh, Regularity of harmonic maps from the Minkowski space into rotationally symmetric manifolds, Comm. Pure Appl. Math. 45 (1992), 947-971.

[16] Fu-Wen Shu and You-Gen Shen, Geometric flows and black holes, arXiv: gr-qc/0610030.

Wen-Rong Dai, De-Xing Kong

Center of Mathematical Sciences, Zhejiang University

Hangzhou 310027, China

E-mail: kong@cms.zju.edu.cn.

Kefeng Liu

Center of Mathematical Sciences, Zhejiang University

Hangzhou 310027, China

or

Department of Mathematics, UCLA, CA 90095, USA

E-mail: liu@math.ucla.edu 